\title{
Hyperpolarization via dissolution dynamic nuclear polarization: new technological and methodological advances
}

Pinon, Arthur César; Capozzi, Andrea; Ardenkjær-Larsen, Jan Henrik

Published in:

Magnetic Resonance Materials in Physics, Biology and Medicine

Link to article, DOI:

10.1007/s10334-020-00894-w

Publication date:

2021

Document Version

Peer reviewed version

Link back to DTU Orbit

Citation (APA):

Pinon, A. C., Capozzi, A., \& Ardenkjær-Larsen, J. H. (2021). Hyperpolarization via dissolution dynamic nuclear polarization: new technological and methodological advances. Magnetic Resonance Materials in Physics, Biology and Medicine, 34, 5-23. https://doi.org/10.1007/s10334-020-00894-w

\section{General rights}

Copyright and moral rights for the publications made accessible in the public portal are retained by the authors and/or other copyright owners and it is a condition of accessing publications that users recognise and abide by the legal requirements associated with these rights.

- Users may download and print one copy of any publication from the public portal for the purpose of private study or research.

- You may not further distribute the material or use it for any profit-making activity or commercial gain

- You may freely distribute the URL identifying the publication in the public portal 


\title{
Hyperpolarization via dissolution Dynamic Nuclear Polarization: new technological and methodological advances
}

\author{
Arthur C. Pinon, ${ }^{1}$ Andrea Capozzi, ${ }^{1,2}$ and Jan Henrik Ardenkjær-Larsen. ${ }^{1, *}$
}

${ }^{1}$ Center for Hyperpolarization in Magnetic Resonance, Department of Health Technology, Technical University of Denmark, Building 349, 2800 Kgs Lyngby, Denmark;

${ }^{2}$ Laboratory for Functional and Metabolic Imaging, École Polytechnique Fédérale de Lausanne, Route Cantonale, 1015 Lausanne, Switzerland.

\begin{abstract}
Dissolution-DNP is a method to boost liquid-state NMR sensitivity by several orders of magnitude. The technique consists in hyperpolarizing samples by solid-state dynamic nuclear polarization at low temperature and moderate magnetic field, followed by an instantaneous melting and dilution of the sample happening inside the polarizer. Although the technique is well established and the outstanding signal enhancement paved the way towards many applications precluded to conventional NMR, the race to develop new methods allowing higher throughput, faster and higher polarization and longer exploitation of the signal is still vivid. In this work, we review the most recent advances on dissolution-DNP methods trying to overcome the original technique's shortcomings. The review describes some of the new approaches in the field, firstly, in terms of sample formulation and properties, secondly, in terms of instrumentation.
\end{abstract}

\section{Key words}

Hyperpolarization, Dynamic Nuclear Polarization, dDNP, non-persistent radicals, cross-polarization, cryogen-free, bullet DNP.

\section{Acknowledgements}

This work was supported by the Danish National Research Foundation (DNRF124); the European Union's Horizon 2020 research and innovation programme under the Marie Sklodowska-Curie grant agreement no. 713683 (COFUNDfellowsDTU); and the Swiss National Fund under the SPARK grant agreement no. CRSK-2_190547.

\section{Corresponding author}

*Prof. Jan Henrik Ardenkjær-Larsen

Technical University of Denmark

Ørsted Plads, Building 349, room 126

2800 Kgs. Lyngby

T: +45 452539 18; Email: jhar@dtu.dk 


\section{Introduction}

Nuclear Magnetic Resonance (NMR) and its two main applications, i.e. Magnetic Resonance Spectroscopy (MRS) and Magnetic Resonance Imaging (MRI), play a crucial part in many different fields ranging from material science, to molecular biology and clinical applications. MRS is considered one of the most powerful analytical techniques due to the detailed information that it can provide about molecular and macromolecular structures, dynamic processes, and chemical reactions in both material and biological studies, to name few. MRI is a medical diagnostic technique characterized by high anatomical resolution and a plurality of image contrast mechanisms; able to highlight, as appropriate, various pathologies and their response to treatment. Moreover, unlike computed tomography (CT) and positron emission tomography (PET), the absence of ionizing radiations makes MRI a completely non-invasive diagnostic technique. Nevertheless, NMR has a main drawback: many of its applications suffer from lack of sensitivity, which translates into long acquisitions and poor temporal resolution. The NMR signal is proportional to the nuclear magnetization $(M)$ that, in turn, increases linearly with the nuclear spin polarization $P_{I}$ :

$$
M=N \mu_{I} P_{I}
$$

where $N$ represents the number of spins per unit volume and $\mu_{I}$ the magnetic moment of the nuclei with spin quantum number $I$. The nuclear polarization describes the population difference among spin energy eigenstates, providing a measurement of the degree of alignment between the net magnetic moment and the external magnetic field $\mathrm{B}_{0}$, conventionally applied along the z-axis. For achievable values of the magnetic fields and at room temperature, the thermal energy is several orders of magnitude larger than the nuclear spins magnetic energy. Consequently, the difference in spin population between adjacent nuclear spin energy eigenstates is extremely small. The latter translates into a weak net magnetic moment and thus poor NMR signal.

At thermal equilibrium the spin energy level population follows a Boltzmann distribution. Said $m_{I}$ the projection of the spin magnetic moment along the z-axis for a given energy eigenstate, the polarization writes [1]:

$$
P_{I}=\frac{1}{I} \cdot \frac{\sum_{m_{I}} m_{I} \exp \left(-E_{m_{I}} / k_{B} T\right)}{\sum_{m_{I}} \exp \left(-E_{m_{I}} / k_{B} T\right)}
$$

In case of spin $\mathrm{I}=1 / 2$ (e.g. ${ }^{1} \mathrm{H}$ and ${ }^{13} \mathrm{C}$ ), only two energy levels are present: $E_{ \pm 1 / 2}= \pm \hbar \gamma B_{0} / 2$. Thus, equation (1) reduces to:

$$
P_{I}=\tanh \left(\frac{\hbar \gamma B_{0}}{2 k_{B} T}\right)
$$

For a magnetic field $\mathrm{B}_{0}$ of $9.4 \mathrm{~T}\left(400 \mathrm{MHz}{ }^{1} \mathrm{H}\right)$ and temperature $\mathrm{T}$ of $298 \mathrm{~K}$, the nuclear spin polarization calculates only $32 \mathrm{ppm}$ for ${ }^{1} \mathrm{H}$ and $8.1 \mathrm{ppm}$ for ${ }^{13} \mathrm{C}$. This means that on average, out of $10^{6}$ nuclear spins, only 32 contribute to the NMR signal in the first case and 8 in the latter case.

Looking at equation (1), the most straightforward way to improve NMR sensitivity would be to increase the nuclear polarization. For a given spin system, equation (3) shows two parameters that can be controlled: the temperature and the magnetic field. Unfortunately, cooling the sample is not often a valuable option since most NMR applications entail working at room temperature. This explains why, over the years, the general approach to circumvent NMR low sensitivity 
has been to develop higher magnetic field strengths; with the highest commercial MRI field strength of $11.75 \mathrm{~T}$ for humans and $21 \mathrm{~T}$ for preclinical use. However, this comes at an exorbitant cost for an improvement in signal to noise of only one order of magnitude. A different and more efficient approach is to "artificially" cool down the sample by breaking the Boltzmann thermal equilibrium and enhancing the population difference between the energy states. All methods able to establish such a non-equilibrium spins distribution are known as hyperpolarization techniques. Currently, the most common techniques include hyperpolarization by means of brute force (BF) [2], spin exchange optical pumping (SEOP) [3], parahydrogen-induced polarization (PHIP) [4] and Dynamic Nuclear Polarization (DNP) [5].

DNP takes advantage of the unpaired electrons spin's high polarization compared to the nuclear spins for the same experimental conditions. Microwave irradiation, close to the electron Larmor frequency, induces the polarization transfer from the electron spins to the nuclei nearby. Discovered in the 1950's by Overhauser, Carver and Slichter [6, 7], the technique was initially used for high energy physics experiments [8], before the magnetic resonance community adopted it for enhancing the sensitivity of both solid-state NMR (MAS-DNP) [9, 10] and liquid-state NMR (Overhauser DNP [11] or dissolution DNP (dDNP) [12]).

Dissolution DNP was invented in 2003 by Ardenkjaer-Larsen et al. [12]. DDNP produces hyperpolarized solutions by dissolving solid samples previously polarized by DNP at liquid helium temperature $(1-1.4 \mathrm{~K})$ and moderate magnetic field (3.35 - $7 \mathrm{~T}$ ), using microwaves irradiation at relatively low power (typically less than $100 \mathrm{~mW}$ ). Unpaired electron spins are present in the sample in the form of organic free radicals. These radicals, broadly defined as electron polarizing agents (EPAs), are usually present at concentrations of the order of tens of millimolars [13]. Generally, the target nuclei during a dDNP experiment have a low- $\gamma\left({ }^{13} \mathrm{C},{ }^{6} \mathrm{Li}\right.$ and $\left.{ }^{15} \mathrm{~N}\right)$ and solid-state concentration in the range $1-10 \mathrm{M}$, since their slower longitudinal relaxation after dissolution extends the available time window for exploiting the hyperpolarized signal [14-19]. More recently, advances in the technique allowed to broaden the focus towards water protons hyperpolarization for chemical and pre-clinical applications [20-24].

There exist three commonly accepted mechanisms to describe the DNP process, depending on the experimental conditions: the solid effect (SE), cross effect (CE) and thermal mixing (TM) [5, 25]. SE is a two-spins process involving an electron spin coupled to a nuclear spin by dipolar interaction. The transfer of the electron spin polarization to the nuclei is induced by microwave irradiation at frequency $v_{\mu \mathrm{w}}=v_{0, \mathrm{e}}-v_{0, \mathrm{n}}$ or $v_{\mu \mathrm{w}}=v_{0, \mathrm{e}}+v_{0, \mathrm{n}}$ (where $v_{0, \mathrm{e}}$ and $v_{0, \mathrm{n}}$ are the electronic and nuclear Larmor frequencies, respectively). Irradiating at $v_{\mu \mathrm{w}}=v_{0, \mathrm{e}}-v_{0, \mathrm{n}}$ saturates the double-quantum (DQ) transition of the electron-nucleus two-spin system. Similarly, irradiation at $v_{\mu \mathrm{w}}=v_{0, \mathrm{e}}+v_{0, \mathrm{n}}$ excites the zero-quantum (ZQ) transition. Such transitions, known as "forbidden" because they violate the selection rules at the first order, represent second-order processes that can take place thanks to the state mixing caused by the non-secular part the dipolar Hamiltonian. As SE involves second order transitions, it requires high power microwaves irradiation because of the relatively low transition probability involved [25-28].

CE and TM belong to a different family of mechanisms whose main feature is the occurrence of a "triple spin flip". CE can be described as a dipolar coupled three-spins system involving one nucleus $n$ and two electrons $\left(e_{1}, e_{2}\right)$ with Larmor frequencies satisfying the relation $v_{0, \mathrm{e} 1}-v_{0, \mathrm{e} 2}=v_{0, \mathrm{n}}$. This is the case when $e_{1}$ and $e_{2}$ have two different molecular orientations resulting in two different resonances. Microwaves irradiation at $v_{\mu \mathrm{w}}=v_{0, \mathrm{e} 1}$, where $v_{0, \mathrm{e} 1}<v_{0, \mathrm{e} 2}$, saturates the electron spin resonance at lower energy triggering energy-conserving flip-flop-flip transitions that increase the nuclear spins population on the lower Zeeman level, thus resulting in positive increase of the nuclear polarization. Conversely, irradiation at $v_{\mu \mathrm{w}}=v_{0, \mathrm{e} 2}$ generates a negative increase of the nuclear spin polarization. In TM, polarization is transferred 
via triple spin flips as well, but it is a multi-body mechanism based on the macroscopic concept of spin temperature, thus requiring a strong coupling between the electron spins [28-31]. In both CE and TM, allowed electron spin transitions are involved. The latter lifts the requirements of high microwave power that is beneficial when working at low temperature. Therefore, when the triple spin flip condition is satisfied, TM and CE are the dominant mechanism at dDNP experimental conditions.

The EPA's molecular structure and couplings between the unpaired electron and neighboring nuclei (hyperfine interaction) determine the electron spin resonance (ESR) spectrum. Working with spin $1 / 2$ radicals and at magnetic field values typical for dDNP, the main contribution to the ESR spectral appearance comes from the Zeeman interaction. In case the molecular magnetic environment is non-homogeneous with respect to the direction of the external field, the ESR resonance position depends on the orientation of the EPA molecule. This phenomenon is the ESR equivalent of the NMR chemical shift anisotropy (CSA) and known as g-anisotropy. Moreover, if the hyperfine interaction has a dipolar component (e.g. nitroxide radials), it will be anisotropic as well. These two anisotropic contributions generate the socalled inhomogeneous broadening of the ESR spectrum. If the EPAs' concentration is high enough, the electron spins will be strongly coupled by dipolar interaction. On average this contribution symmetrically broadens each ESR line of the spectrum generating the so-called homogeneous broadening. The relative magnitudes of the homogeneous vs the inhomogeneous broadening has implications on the mechanism leading to the nuclear polarization enhancement: CE vs. TM [32].

\section{Original sample formulation}

In its original form, the DNP sample is made of three components: the substrate molecules to be hyperpolarized, an EPA, and a glassing agent if the substrate is not self-glassing when frozen. The role of the glassing agent is to ensure a homogeneous distribution of the EPAs. Indeed, a crystallization of the matrix would segregate the EPAs to the boundaries of the crystalline domains, leading to inefficient DNP [33]. The concentration of the substrate is usually on the molar range, while the EPA concentration is usually on the order of millimolar. One of the most important characteristics of an EPA for an efficient DNP process is its ESR linewidth. Since at low microwave powers CE and TM mechanisms are the dominant DNP mechanisms, the ESR linewidth has to be larger than the Larmor frequency of the nucleus of interest for triple spin-flips to take place. Therefore, if the aim is to polarize high-gamma nuclei such as ${ }^{1} \mathrm{H}$ or ${ }^{19} \mathrm{~F}$, the ESR linewidth has to be hundreds of $\mathrm{MHz}$ large, which is typically the case for nitroxide radicals [34]. Differently, if one desires to polarize low-gamma nuclei such as ${ }^{13} \mathrm{C},{ }^{15} \mathrm{~N}$ or ${ }^{6} \mathrm{Li}$, it is preferable to use an EPA with ESR linewidth larger than their Larmor frequency, but smaller than the solvent abundant ${ }^{1} \mathrm{H}$ nuclei Larmor frequency. Indeed, the "polarization power" of the EPA is shared among all nuclear species in the sample. This explains why Trityl and BDPA radicals are two of the most efficient EPAs to hyperpolarize low-gamma nuclei [34].

\section{Original DNP polarizer}

In the seminal work by Ardenkjær-Larsen et al. [12], DNP was achieved on a static sample at low temperatures and moderate magnetic field. The DNP polarizer presented three main components. First, a cold bore superconductive magnet working at $3.35 \mathrm{~T}$ and at temperatures ranging from $1.2 \mathrm{~K}$ to $4.2 \mathrm{~K}$, ensuring high enough electron Boltzmann polarization ( $95 \%$ at this experimental conditions); second, a microwave source coupled to a rectangular waveguide to irradiate the sample sitting in liquid helium and placed in the isocenter of the magnet; third, a dissolution system able to melt the 
sample inside the polarizer while keeping the hot buffer isolated from the cryogenic fluids. A sketch of the original dDNP polarizer design developed by Ardenkjær-Larsen et al. is presented in Fig. 1.

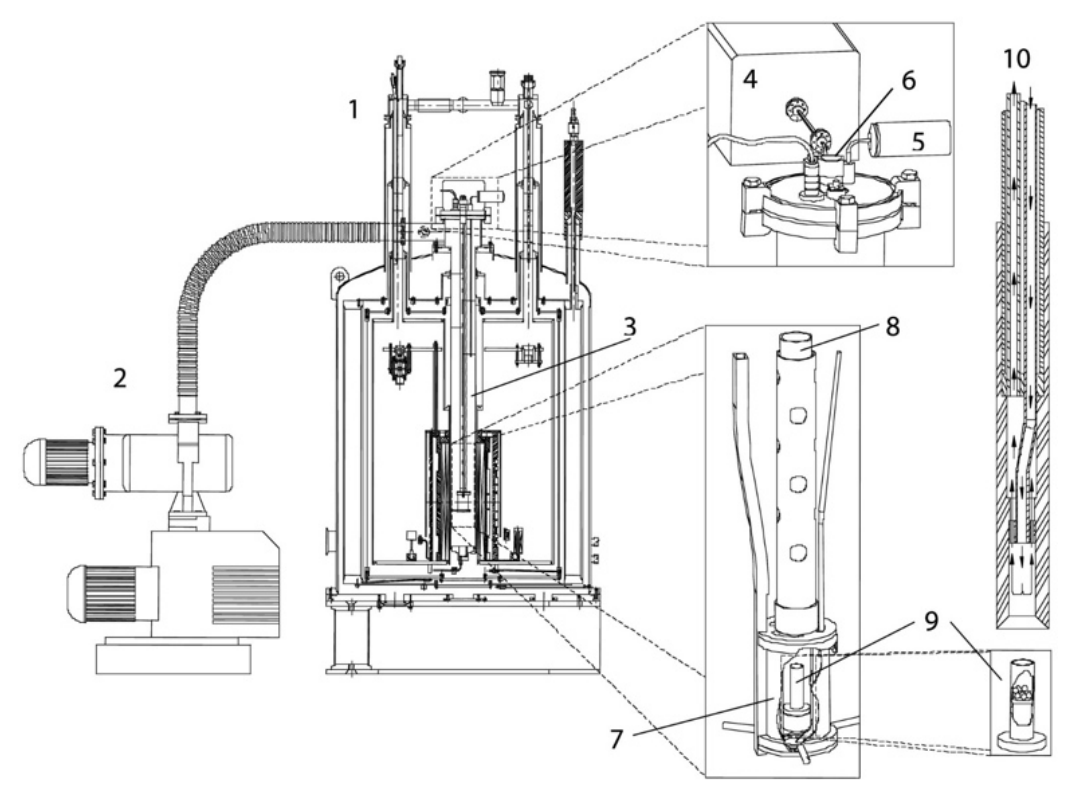

Fig. 1. Schematic drawing of the original dDNP polarizer developed by Ardenkjær-Larsen et al:: (1) DNP polarizer, (2) vacuum pumps, (3) variable temperature insert (VTI), (4) microwave source, (5) pressure transducer, (6) sample port, (7) microwave cavity, (8) sample holder, (9) sample container and (10) dissolution wand. Drawing taken from ref [12].

Performing dDNP at a low temperature is crucial, not only because the unpaired electron spin polarization represents the highest limit the nuclear spins can achieve, but also for relaxation times arguments. Indeed, at experimental conditions typical for dDNP, the electron spin-lattice relaxation time $\left(\mathrm{T}_{1 \mathrm{e}}\right)$ is long enough (hundreds of milliseconds [35]) to ensure efficient microwave saturation of the ESR line. The latter is a crucial condition for an efficient DNP process [5]. Increasing the magnetic field yields to higher nuclear polarization, but also increases the build-up time [36]. Increasing the microwave power decreases the polarization build-up time, but also increases sample heating [37]. Although the commercially available SPINlab ${ }^{\mathrm{TM}^{*}}$ polarizer by General Electric (Milwaukee, WI, USA) choice was to work at $5 \mathrm{~T}$ and $0.8 \mathrm{~K}$ [38], a field of 6.7-7 $\mathrm{T}$ and a temperature of 1.2 $-1.4 \mathrm{~K}$ seem to be a better compromise in terms of complexity of the cryogenic machinery and achievable polarization in a reasonable amount of time [39]. To date, the best methods to maximize the solid-state polarization are as follows. If the target nuclei have a low-gamma (e.g. ${ }^{13} \mathrm{C},{ }^{6} \mathrm{Li}$, $\left.{ }^{15} \mathrm{~N}\right)$, employing narrow ESR line radicals (e.g. trityl and BDPA) with the addition of small amounts $(1-2 \mathrm{mM})$ of a gadolinium complex represents the best strategy [40-42]. Conversely, if high-gamma nuclei are involved (e.g. $\left.{ }^{1} \mathrm{H}\right)$ employing broad ESR line radicals (e.g. nitroxides) together with microwave frequency modulation provides better performance [20, 43-45]. Crosspolarization and microwaves gating have been employed to transfer high spin order from protons to low-gamma nuclei in a reduced amount of time and employing broad ESR line radicals [37, 46].

\section{$\underline{\text { Original dissolution procedure }}$}

Once the nuclear polarization has reached its maximum value, dissolution is performed by rapidly melting the frozen sample with a hot solvent. The resulting hyperpolarized solution is then chased out of the DNP polarizer using pressurized helium gas and collected for in vivo or in vitro applications. The key challenge of the dissolution procedure is to preserve as much nuclear polarization as possible with respect to the solid-state value. 
This task is challenging because nuclear spin-lattice relaxation $\left(\mathrm{T}_{1, \mathrm{n}}\right)$ in the solid-state at high temperatures and low magnetic field is prohibitively short (i.e. milliseconds) in presence of paramagnetic centers. Thus, the dissolution has to happen inside the polarizer in a high magnetic field, but above liquid helium level for efficient heat transfer to the sample. To this hand, employing high heat capacity solvents (e.g. water) and freezing the sample as small pellets can ease the procedure [47]. Fast transfer, removal of the EPAs and use of a magnetic tunnel [48] are also beneficial to shelter the nuclear polarization across the path between the polarizer and the NMR magnet.

\section{Shortcomings of the original techniques}

However, the original procedure has several shortcomings. Firstly, besides the special case of pyruvic acid, the substrate of interest is often mixed with a glassing agent to insure homogeneous distribution of the radicals in the frozen sample matrix. The presence of glassing agents dilutes the analyte and can represents a limitation for in vivo applications. Secondly, the permanent radicals employed in the traditional sample formulation preclude the possibility to extract the hyperpolarized sample in the solid-state from the polarizer to be stored at cryogenic temperature and appropriate magnetic field values to be transported to a remote location. This shortcoming has a main implication: the dDNP machine has to be placed as close as possible to the measuring apparatus, making unlikely to equip each MR facility with hyperpolarization because of the high cost and demanding technical skills required. Thirdly, the sample is highly diluted (10 - 50 times) during the dissolution step, causing severe limitations to applications concerning small volumes. Fourthly, the short lifetime of hyperpolarization after dissolution limits the signal exploitation to a time window of maximum one minute. Lastly, liquid helium is a limited and costly resource and a traditional "wet" polarizer needs between 50 and 100 L per week to run continuously.

In the following we will review new approaches to tackle these challenges.

\section{New dDNP methods: alternative sample formulation}

\section{$\underline{\text { DNP without glassing agent }}$}

\section{Vitrified droplets methods}

Because of the undisputed usefulness in biomedical application, high achievable polarization and relatively long liquidstate $\mathrm{T}_{1, \mathrm{n}},\left[1-{ }^{13} \mathrm{C}\right]$ pyruvic acid doped with trityl free radicals has become the most studied and used dDNP sample [4951]. However, a major challenge in extending the range of metabolomic experiments is to find biologically compatible mixtures in which an efficient EPA and the metabolic substrate of interest can be co-dissolved easily and remain well mixed upon freezing. In 2015, Joanna Long et al. explored an alternative method of sample preparation that does not involve any glassing agent [52].

Usually, the dDNP sample preparation is performed by freezing an aqueous solutions in liquid nitrogen to form a glassy matrix [47]. However, due to the inverse Leidenfrost effect, liquid nitrogen suddenly transforms to gas on contact with the sample. The result is the appearance of an insulating layer of nitrogen gas that transfers heat at a lower rate than liquid nitrogen. The consequent slower freezing of the sample can cause segregation of the radicals and thus poor DNP [53, 54]. For this reason, it is common to employ glassing agents that prevent crystallization when the sample is frozen in liquid nitrogen. Common glassing agents are ethanol (EtOH), glycerol or dimethyl sulfoxide (DMSO). The method employed 
by Long et al. consisted in spraying as a fine mist a liquid mixture containing the stable radical, a non-glassing solvent and the labelled metabolite, in a cold liquid alkane solution, typically isopentane (Fig. 2a). Since liquid alkanes are known to have a better heat transfer efficiency than liquid nitrogen [55], isopentane was employed and cooled down to $130 \mathrm{~K}$. This technique allowed the formation of small vitrified droplets. The frozen droplets typically produced by this method were not a perfectly amorphous, but still glassy enough to sustain a pseudo-homogeneous distribution of molecules and ensure an efficient DNP process. By comparing a substrate/radical mixture with and without ethanol (EtOH) as glassing agent, and using cold nitrogen or isopentane as freezing media, it was possible to test the efficiency of this method with respect to a typical standard DNP sample preparation.
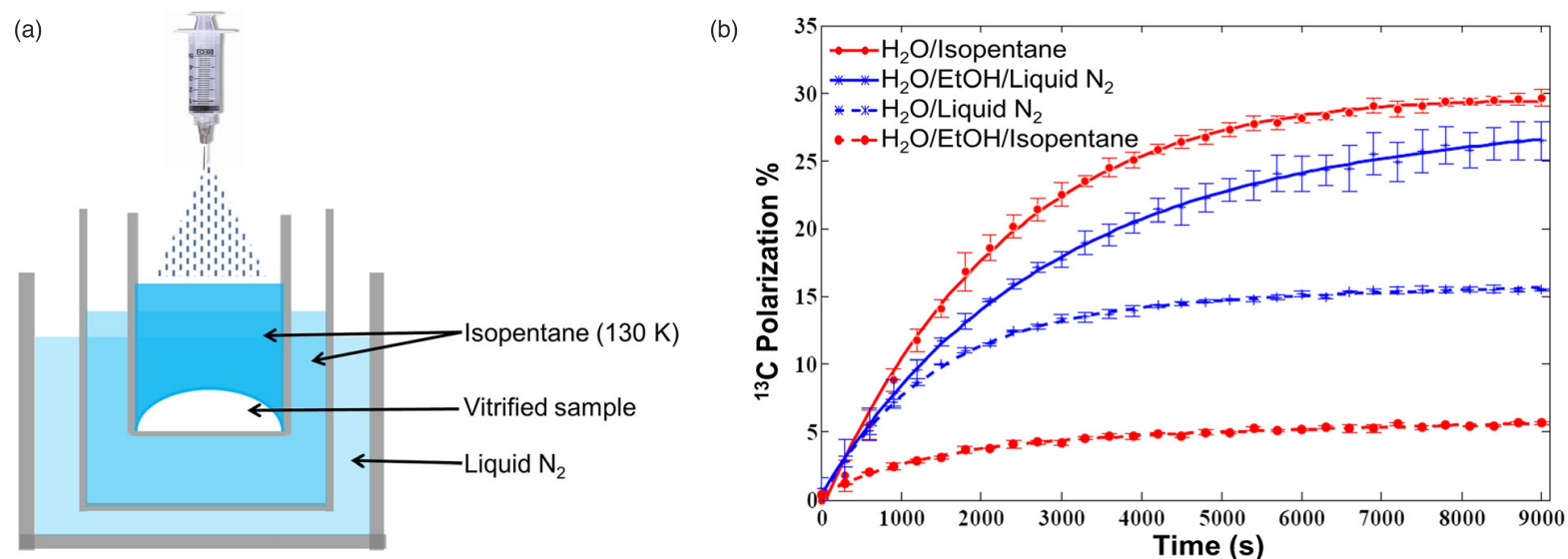

Fig. 2. (a) Schematic drawing of the set-up used to freeze aqueous solutions into vitrified droplets. (b) Polarization build-up curves at $5 \mathrm{~T}$ and $\leq 1.2 \mathrm{~K}$ of aqueous solutions containing $3.0 \mathrm{M}$ of sodium $\left[1-{ }^{13} \mathrm{C}\right]$ acetate and $15 \mathrm{mM}$ trityl OX063 with or without ethanol as co-solvent, and frozen in isopentane (red) or in liquid nitrogen (blue). Fig. adapted from ref [52].

As shown in Fig. 2b, Long et al. achieved a ${ }^{13} \mathrm{C}$ polarization of $29 \%$ when dissolving sodium $\left[1-{ }^{13} \mathrm{C}\right]$ acetate and trityl in water (without ethanol) and freezing it in isopentane, with a build-up time constant of $2300 \mathrm{~s}$. Other parameters being identical, the ${ }^{13} \mathrm{C}$ polarization was lower when the sample was frozen with nitrogen instead of isopentane. This was due to a higher heat transfer efficiency of isopentane resulting in a better glass formation. However, when ethanol was used as glassing agent, the polarization was higher when freezing the sample in liquid nitrogen instead of cold isopentane. This was a consequence of the ethanol melting temperature $(159 \mathrm{~K})$. Using ethanol as co-solvent considerably lowered the freezing point of the mixture and, most likely, the isopentane temperature of $130 \mathrm{~K}$ prevented from making a stable glass [56]. Once placed into the helium cryostat, the sample probably had a further phase transition from a very viscous solution to an actual solid resulting in a heterogeneous distribution of radicals and metabolic substrate and, thus, a poor DNP efficiency.

All measured ${ }^{13} \mathrm{C}$ polarizations were lower than using neat pyruvic acid with trityl $\left(\mathrm{P}\left({ }^{13} \mathrm{C}\right)=60 \%\right)$, since the matrix, not optimized here, plays a crucial role in achieving high polarization values $[47,57]$. Polarization build-ups (see Fig. 2b) were comparable to the ones typically measured at this field and temperature on standard sample preparations. Build-up times were similar with and without ethanol as a glassing agent, since the ${ }^{1} \mathrm{H}$ spin concentration did not have a large effect on the spin temperature of the matrix [58]. A similar result was obtained with sodium $\left[1-{ }^{13} \mathrm{C}\right]$ acetate and $50 \mathrm{mM}$ of $4-$ oxo-TEMPO in deuterated water: a ${ }^{13} \mathrm{C}$ polarization of $28 \%$ was reached, while yielding a shorter build-up time of $480 \mathrm{~s}$ due to a higher radical concentration. Dissolutions were performed on samples mixed with TEMPOL, leading to liquid- 
state ${ }^{13} \mathrm{C}$ polarizations of approx. $6 \%$ at $4.7 \mathrm{~T}$. High polarization losses were observed during dissolution and transfer, mostly as a result of a long transfer time $(15 \mathrm{~s})$. The high 4-oxo-TEMPO concentration reduced $\mathrm{T}_{1}$ after dissolution and lead to significant loss of polarization during the transfer.

The method shows that it is possible to obtain high solid-state polarizations with significant liquid-state enhancement after dissolution although no glassing agent is involved. The method is applicable for a large range of metabolic substrates, alleviating toxicity problems in particular for in vivo metabolic studies.

\section{Thermo-responsive agents}

Removing radicals from the HP solution is a key requirement for prolonging the hyperpolarization life-time and when performing in vivo applications. An alternative approach with respect to the time consuming chemical scavenging or phase separation $[23,59]$ is to use labelled polymers that precipitates at specific experimental conditions. In 2016, Cheng et al. showed that tert-butanol could be polarized using radical-bearing thermo-responsive polymers (hydrogel). At room temperature, the polymers can be impregnated with a solution containing the substrate of interest. During dissolution, when the temperature increases above $63{ }^{\circ} \mathrm{C}$, the polymers collapse, expelling the HP substrate and segregating the radicals. This technique allowed an increase of the hyperpolarization life-time by a factor four in the liquid-state [60]. In a similar way, Vuichoud et al. hyperpolarized water protons and metabolites using TEMPO moieties grafted to another thermo-responsive polymer (FLAP) that precipitates and can be easily filtered when the temperature increases above 32 ${ }^{\circ} \mathrm{C}$ in the presence of $\mathrm{NaCl}[61]$.

\section{Silica backbones methods}

Using EPAs grafted to the surface of silica-based nanoporous materials offers a way to combine together easy radicals removal and absence of glassing agent for sample preparation [62]. The porous material is impregnated with a substratecontaining solution, frozen and loaded inside the DNP polarizer. By shining microwaves, the EPAs polarize the substrate that entered the pores. Since the radical distribution is pre-determined by the silica backbone structure, the substratecontaining solution may crystallize without changing the distribution of the EPAs. Moreover, the silica backbones can be easily filtered during dissolution [61], leaving a radical free HP liquid [59]. Yet, for the hyperpolarization to survive, this method still requires the dissolution to happen inside the polarizer. The EPAs are close enough to the substrate of interest (few nanometers) to preclude extraction of the HP sample in the solid state with the aim of storage and transport of the latter to a remote location. After dissolution the HP state lifetime is limited to the minute scale, imposing to place each polarizer in close proximity to each NMR magnet/MRI scanner. Hyperpolarization non-transportability is for sure a shortcoming. To tackle this problem, two methods recently emerged: 1) the use of microcrystalline solids [63] and 2) the use of labile radicals $[64,65]$.

\section{Transportable hyperpolarization}

\section{Dissolution-DNP using microcrystalline solids}

The concept of using microcrystalline solids for dDNP is to physically separate the EPAs and the substrate on a micrometer length scale. The concept is not new, since it was first used by Griffin et al. in magic angle spinning DNP to hyperpolarize amyloid fibrils in 2006 [66]. Later on, Emsley et al. used the same idea to determine the structure of 
microscopic domains $[67,68]$. The technique consisted in grinding a crystalline solid (e.g. sodium $\left[1-{ }^{13} \mathrm{C}\right]$ pyruvate) to form microcrystalline particles. Then, the particles were impregnated with a radical-containing solution. The liquid component of the solution was chosen for not dissolving the crystalline solid and obtain a heterogeneous liquid-solid sample after impregnation. The wet mixture was then frozen, creating two solid phases: the radical-containing frozen solution that included ${ }^{1} \mathrm{H}$ spins and unpaired electron spins, and the microcrystalline solid which contained ${ }^{1} \mathrm{H}$ and ${ }^{13} \mathrm{C}$ spins with no radicals. During microwave irradiation, the proton spins from the radical solution were rapidly polarized. Then, due to a polarization gradient between the frozen solution and the microcrystalline solid, the hyperpolarization was spontaneously transferred to the inside of the particle by ${ }^{1} \mathrm{H}-{ }^{1} \mathrm{H}$ spin diffusion. The time required to polarize the crystalline solid was usually longer compared to standard DNP frozen solutions [69].

(a)

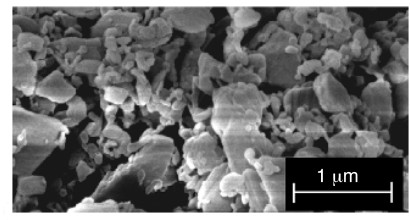

SEM of $\left(1-{ }^{13} \mathrm{C}\right)$ sodium pyruvate
$\Rightarrow$

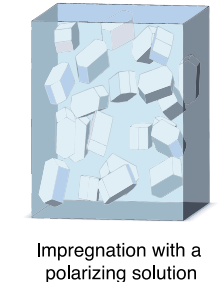

(b)
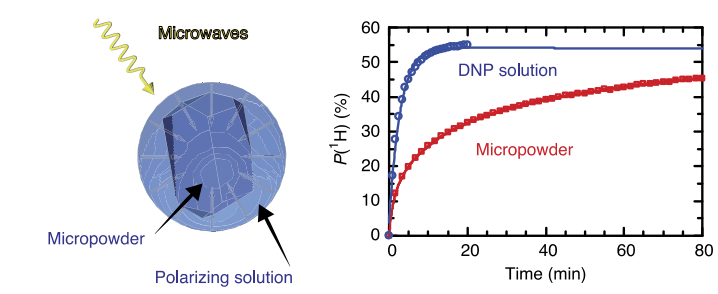

(d)

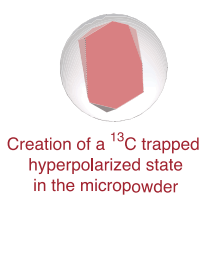

(c)

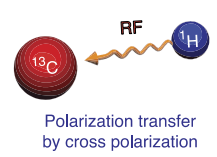

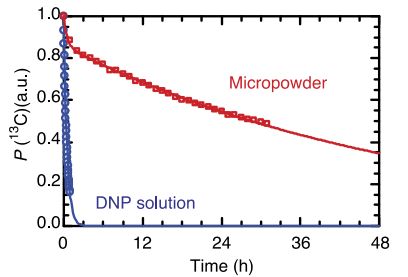

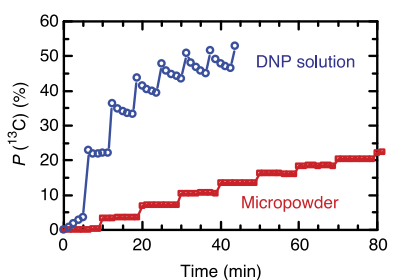

Fig. 3. Illustration of the steps for hyperpolarizing micro particles. (a) A grounded powder of sodium $\left[1-{ }^{13} \mathrm{C}\right]$ pyruvate was impregnated with toluene$d_{6}$ :THF- $d_{8}$ :THF (8:1:1). The sodium pyruvate was insoluble in the organic solvent. (b) ${ }^{1} \mathrm{H}$ DNP build-up at $6.7 \mathrm{~T}$ and $1.2 \mathrm{~K}$ (blue) of a mixture of sodium $\left[1-{ }^{13} \mathrm{C}\right]$ pyruvate in $\mathrm{H}_{2} \mathrm{O}: \mathrm{D}_{2} \mathrm{O}$ :glycerol- $d_{8}(2: 3: 5)$ with $40 \mathrm{mM}$ TEMPOL, and (red) ${ }^{1} \mathrm{H}$ DNP build-up of powdered sodium $\left[1-{ }^{13} \mathrm{C}\right]$ pyruvate impregnated with toluene- $d_{6}$ :THF- $d_{8}$ :THF (8:1:1) with $40 \mathrm{mM}$ TEMPOL-benzoate. (c) ${ }^{13} \mathrm{C} \mathrm{CP-DNP}$ build-up curves of the radical solution (blue) and the solid (red) using ${ }^{1} \mathrm{H}-{ }^{13} \mathrm{C}$ cross polarization. (d) Decay of the ${ }^{13} \mathrm{C}$ signal at $4.2 \mathrm{~K}$ and $6.7 \mathrm{~T}$ in the radical solution (blue) and the solid (red). Fig. reproduced from ref [63].

In Fig. $3 b$ (blue circles) is shown the ${ }^{1} \mathrm{H}$ DNP build-up at $1.2 \mathrm{~K}$ and $6.7 \mathrm{~T}$ of sodium $\left[1-{ }^{13} \mathrm{C}\right]$ pyruvate in $\mathrm{H}_{2} \mathrm{O}: \mathrm{D}_{2} \mathrm{O}: \mathrm{glycerol}-$ $d_{8}(2: 3: 5)$ with $40 \mathrm{mM}$ TEMPOL, quickly reaching a polarization higher than $50 \%$ and showing a mono-exponential build-up $\left(\operatorname{t~}_{\mathrm{DNP}}\left({ }^{1} \mathrm{H}\right)=150 \mathrm{~s}\right)$ characteristic of a homogeneous distribution of EPAs. Differently, Fig. $3 \mathrm{~b}$ (red circles) shows the ${ }^{1} \mathrm{H}$ DNP build-up of powdered sodium $\left[1-{ }^{13} \mathrm{C}\right]$ pyruvate impregnated with toluene- $d_{6}:$ THF- $d_{8}:$ THF $(8: 1: 1)$ and doped with $40 \mathrm{mM}$ of TEMPOL-benzoate. It can be observed that the ${ }^{1} \mathrm{H}$ DNP build-up curve followed an exponential build-up with two main components. A fast component $t_{D N P, \text { fast }}\left({ }^{1} \mathrm{H}\right)=155 \mathrm{~s}$ arising from protons within the radical-rich part of the sample, and a slow ${ }^{1} \mathrm{H}^{-1} \mathrm{H}$ spin diffusion driven component $t_{\mathrm{DNP} \text {,slow }}=1900 \mathrm{~s}$ arising from protons within the radical-free parts of the sample. It is worth noting that TEMPO-like molecules with broad ESR lines were used since the targeted spins for the DNP process were protons.

Once the polarization had begun to spread in the radical-free parts, ${ }^{1} \mathrm{H}$ spin order was transferred to the nearby ${ }^{13} \mathrm{C}$ spins of pyruvate molecules by ${ }^{1} \mathrm{H}-{ }^{13} \mathrm{C}$ cross polarization $(\mathrm{CP})[46,70]$. Fig. $3 \mathrm{c}$ shows the comparison between a ${ }^{13} \mathrm{C}$ build-up in either dissolved or powdered sodium $\left[1-{ }^{13} \mathrm{C}\right]$ pyruvate with multiple $\mathrm{CP}$ contacts at $1.2 \mathrm{~K}$. It can be observed that the time between two consecutive $\mathrm{CP}$ contacts was longer in the heterogeneous case due to the slower ${ }^{1} \mathrm{H}$ polarization buildup. 
Moreover, the red curve in Fig. $3 \mathrm{c}$ shows almost no ${ }^{13} \mathrm{C}$ relaxation between CP contacts because of the absence of EPAs placed in close vicinity to ${ }^{13} \mathrm{C}$ spins and EPAs. Therefore, the main mechanism for ${ }^{13} \mathrm{C}$ polarization and relaxation was proton-driven ${ }^{13} \mathrm{C}-{ }^{13} \mathrm{C}$ spin diffusion (PDSD) that spreads the polarization from the surface of the particle to the core and vice versa $[71,72]$. As a result, the ${ }^{13} \mathrm{C}$ polarization reached a lower level in a longer time for the powder sample compared to the standard sample, but in turn the spin-lattice relaxation time was considerably longer in the first case. The sodium $\left[1-{ }^{13} \mathrm{C}\right]$ pyruvate $\mathrm{T}_{1}\left({ }^{13} \mathrm{C}\right)$ at $4.2 \mathrm{~K}$ and $6.7 \mathrm{~T}$ for amorphous glass sample and the powder sample measured $20 \mathrm{~min}$ and 37 $\mathrm{h}$, respectively.

The physical separation between the radicals and the ${ }^{13} \mathrm{C}$ nuclear spins can pave a way towards hyperpolarization transport. Indeed Jannin et al. were able to extract the solid sample from the polarizer, while keeping the DNP enhancement alive, and store it for 16h placing it inside a Halbach magnet of 1.0 T immersed in liquid helium.

A more optimized system was developed later by Copéret et al. Here, mesoporous silica materials containing nitroxide radicals were used as " ${ }^{13} \mathrm{C}$ nuclei polarizing network" [73]. In this case the performance was superior tanks to the reduced size of the pores ( 5 to $9 \mathrm{~nm}$ diameter) which enabled a fast ${ }^{1} \mathrm{H}$ build-up, and therefore efficient cross-polarization to ${ }^{13} \mathrm{C}$ nuclei.

\section{Dissolution-DNP using labile radicals}

Another approach to tackle the problem of transportable hyperpolarization is to eliminate the paramagnetic centers from the sample after the DNP process has happened. Non-persistent radicals for dDNP were first introduced in 2013 by Comment et al. [65].The method was first demonstrated using pyruvic acid (PA) as radical precursor [65, 74, 75]. Later, other more optimized precursors were investigated [76, 77]. The radicals are formed by shining UV light on a frozen sample containing a fraction of PA or PA derivatives. By carefully choosing the UV-light wavelength and power, sufficient radicals $(40-50 \mathrm{mM})$ can be generated to perform efficient DNP at high magnetic field [75]. All UV-induced non-persistent radicals share the property to recombine in diamagnetic species for a temperature higher that $190 \mathrm{~K}$ [64]. Since most solvents and substrates used in dDNP sample preparation melt around $273 \mathrm{~K}$, this unique feature allowed the pioneers of the technique to scavenge the radicals inside the dDNP machine, while keeping the substrate still frozen and hyperpolarized. The radicals' annihilation was performed by quickly heating up the sample blowing He gas on it. The absence of paramagnetic species, together with a dramatic increase of the ${ }^{13} \mathrm{C}$ relaxation time, made it possible to quickly extract the sample and melt it outside from the dDNP polarizer, to obtain an HP solution containing $\left[1-{ }^{13} \mathrm{C}\right]$ pyruvate [64]. Although hyperpolarization transport via non-persistent radicals is still in its infancy, a more straightforward advantage of using those EPAs is to obtain a radial free HP liquid after dissolution. The latter is of crucial importance when working with fast relaxing nuclei such as water protons. In the following we will describe more in detail how to perform a dDNP experiment using UV-induced non-persistent radicals and how the precursor molecular structures affects the radical ESR spectrum.

The sample solution must include the radical precursor, the substrate to be polarized and a glassing agent unless the substrate already holds this property. Some droplets of the sample solution are poured in liquid nitrogen, in order to form frozen beads of about $2 \mathrm{~mm}$ diameter. The beads are then transferred to a quartz Dewar for UV light irradiation ( 3 to 10 minutes, depending on precursor concentration and source power). The UV light has the effect of converting a small fraction (1 to $10 \%$ ) of the precursor molecules into ketyl-radicals $[65,75,76]$. The unpaired electron is localized on the $\mathrm{C}_{2}$ atom of the precursor molecule, and is stable as long as the temperature of the sample remains below $190 \mathrm{~K}$. Once the 
radicals are generated, the irradiated sample are transferred to the DNP polarizer keeping it in liquid nitrogen, and quickly loaded. Afterwards, the dDNP experiment follows as usual with the difference that, during dissolution, the sudden temperature jump quenches the EPAs. This fast recombination prevents the need of radicals filtering and most of all relaxation to occur during the dissolution step.
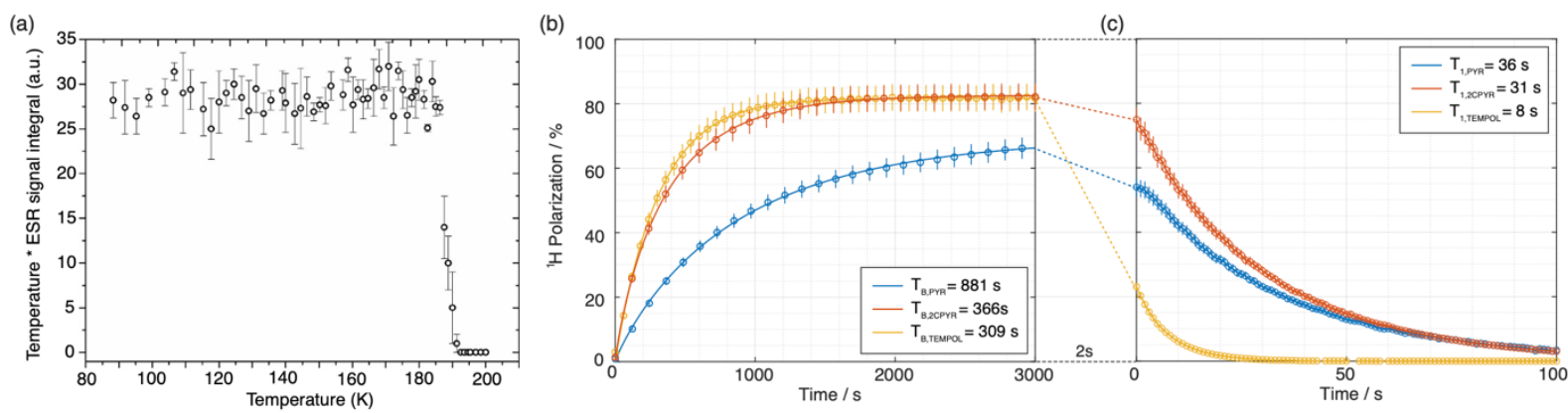

Fig. 4. (a) ESR signal integral multiplied by temperature of frozen PA as a function of temperature. Taken from ref [64]. (b) Solid-state ${ }^{1} \mathrm{H}$ polarization build-up at $6.7 \mathrm{~T}$ and $1.15 \mathrm{~K}$ of PA:glycerol- $d_{8}: \mathrm{H}_{2} \mathrm{O}(2: 3: 5)$ (blue), $\left[2-{ }^{13} \mathrm{C}\right] \mathrm{PA}:$ glycerol- $d_{8}: \mathrm{H}_{2} \mathrm{O}(2: 3: 5)$ (orange), and glycerol- $d_{8}: \mathrm{H}_{2} \mathrm{O}(5: 5)$ with $50 \mathrm{mM}$ of TEMPOL (yellow). (c) Liquid-state relaxation at 9.4 T and $313 \mathrm{~K}$ after dissolution and transfer for the three samples. Taken from ref [20].

The choice of the precursor is functional to the target nuclear species involved. Trimethyl PA and phenyl PA display a narrower ESR line than PA due to a reduced g-anisotropy and hyperfine coupling between the unpaired electron and the methyl group ${ }^{1} \mathrm{H}$ nuclei. This feature was used to highly polarize ${ }^{13} \mathrm{C}[76,77]$. Conversely, $\left[2-{ }^{13} \mathrm{C}\right] \mathrm{PA}$ displays a broader ESR line than PA due to the strong coupling between the unpaired electron and the $\mathrm{C} 2$ carbon. Therefore, the nitroxide radical-like spectrum of UV-irradiated $\left[2-{ }^{13} \mathrm{C}\right] \mathrm{PA}$ together with the fast recombination of the radicals upon dissolution were key features to obtain close to unity water proton polarization [20]. To this regard, in Fig. 4b we report polarization kinetics when using labile radicals to hyperpolarize water protons in a mixture of deuterated glycerol and water at $6.7 \mathrm{~T}$ and $1.15 \mathrm{~K}$. The DNP efficiency was similar for TEMPOL and $\left[2-{ }^{13} \mathrm{C}\right] \mathrm{PA}$. This was due to the fact that both radicals displayed broad enough ESR lines and sufficient concentration to polarize proton spins via TM. The lower efficiency of UV-irradiated PA was due to its narrower ESR line and lower radical concentration. The sudden recombination of the UV-radicals upon dissolution reduced the ${ }^{1} \mathrm{H}$ polarization loss from $70 \%$, when using the stable radical TEMPOL, to only $25 \%$, when using UV-irradiated $\left[2-{ }^{13} \mathrm{C}\right] \mathrm{PA}$. This was confirmed from the fact that sheltering the polarization with a magnetic tunnel was of no influence in the latter case [76, 77].

\section{$\underline{\text { Long-Lived States }}$}

We just discussed the issue of transporting hyperpolarization far away from the production site. Although being able to do so would considerably broaden the HP MR scope, after dissolution the acquisition time window is limited by the intrinsic liquid-state $T_{1 n}$ of the substrate rather than the presence of radicals. For instance, when using trityls as EPAs, the difference in liquid-state $T_{1 n}$ of a substrate with or without radicals in the final solution is negligible. In 2004, Levitt et al. published two breakthrough papers demonstrating that storing nuclear spin order in solution was possible for a time period much longer than $T_{1 n}$. These states are called long-lived states (LLS). [78, 79] Both papers involved molecular systems containing well-isolated pairs of protons in magnetically near-equivalent sites. 
Understanding the magnetic spin states of a $\mathrm{H}_{2}$ molecule can help understanding the basic principle of this technique. A $\mathrm{H}_{2}$ molecule has four magnetic energy eigenstates: three triplet states and one singlet state, depending on the spin up and spin down combinations of the nuclear spin eigenstates. The two ${ }^{1} \mathrm{H}$ spins can be both parallel aligned along the $\mathrm{z}$-axis or against the $\mathrm{z}$-axis forming the $\mathrm{T}_{+1}$ and $\mathrm{T}_{-1}$ triplet states, respectively. If the two spins have opposite orientations, but the wave function is symmetric the pair forms the $\mathrm{T}_{0}$ triplet state. Differently, if the two spins have opposite orientations, and the wave function is antisymmetric the pair forms the $\mathrm{S}_{0}$ singlet state, which is non-magnetic. Both experiments mentioned above exploited a type of nuclear spin order called singlet order, which consists of the difference between the population of the singlet state and the mean population of the three triplet states of proton pairs. Whereas the longitudinal relaxation rate constant $\mathrm{T}_{1}^{-1}$ is dominated by dipole-dipole interactions, the advantageous characteristic of the singlet order relaxation rate constant is that it is immune to such dipolar interactions.

In 2009, Bodenhausen et al. [80] designed a method to preserve the dDNP enhanced magnetization by conversion into long-lived states. They showed that the polarization of natural-abundance ${ }^{13} \mathrm{C}$ spins in a dipeptide could be enhanced directly by a factor close to 10,000 using dDNP, and converted into long-lived states associated with protons, yielding a lifetime seven times longer than the longitudinal relaxation time constant $\mathrm{T}_{1}$ of the same protons not in a singlet state.

The involved experiment comprised five steps (Fig. 5). (1) During the first step, the ${ }^{13} \mathrm{C}$ hyperpolarization was generated at moderate magnetic field and low temperature $\left(\mathrm{B}_{0}=3.35 \mathrm{~T}\right.$ and $\left.\mathrm{T}=1.2 \mathrm{~K}\right)$. After the polarization step, the dissolution step allowed the polarized sample to be transferred as a liquid to the NMR spectrometer $\left(\mathrm{B}_{0}=7.05 \mathrm{~T}\right.$ and $\left.\mathrm{T}=300 \mathrm{~K}\right)$. (2) Then, the polarization was transferred from ${ }^{13} \mathrm{C}$ to ${ }^{1} \mathrm{H}$ using a reverse INEPT. (3) Finally, the ${ }^{1} \mathrm{H}$ polarization was (4) transformed into a long-lived state, and (5) read out by partially converting the LLS into observable proton magnetization. The natural abundance dipeptide Ala-Gly was used for this study. It is worth noting that ${ }^{13} \mathrm{C}$ spins were used as hyperpolarization reservoir during the transfer of the HP solution from the polarizer to the measuring apparatus because of the longer $\mathrm{T}_{1}$ compared to protons.

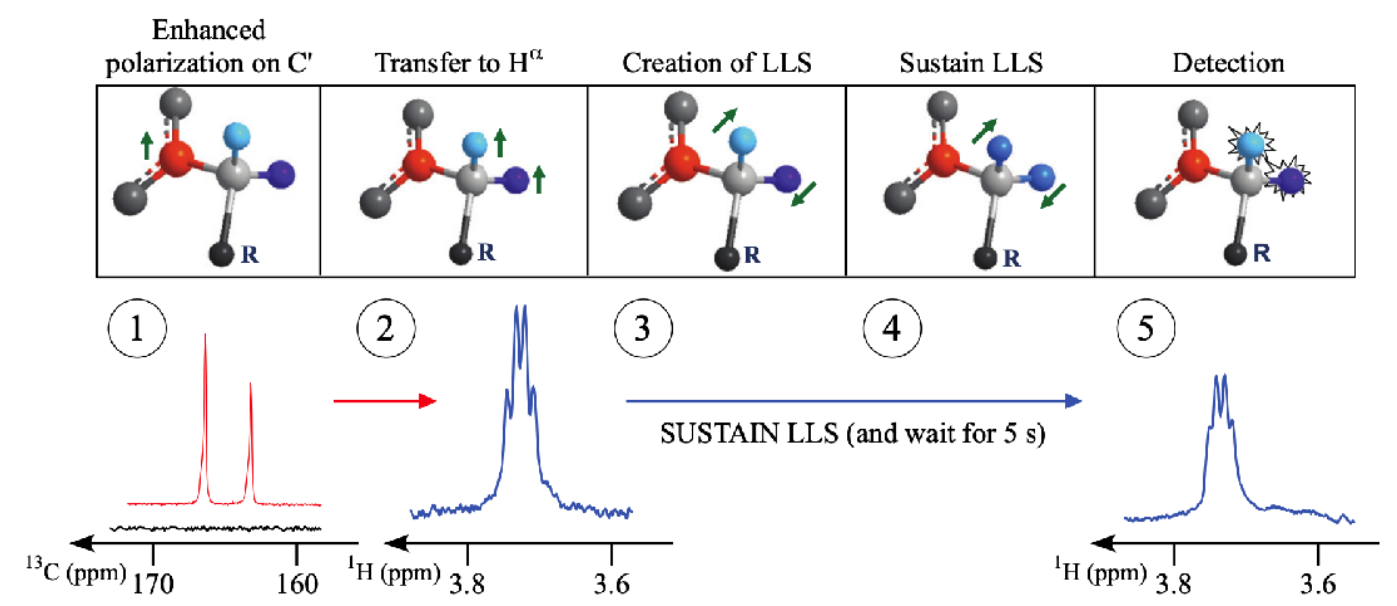

Fig. 5. (1) Hyperpolarized and thermal equilibrium ${ }^{13} \mathrm{C}$ spectra of Ala-Gly. (2) ${ }^{1} \mathrm{H}$ spectrum of the two Gly-H $\mathrm{H}^{\alpha}$ spins in Ala-Gly (blue bowls). (3) Conversion of the magnetization into a LLS. (4) preservation of the LLS on the ${ }^{1} \mathrm{H}$ spins using WALTZ irradiation. (5) Detection of the two proton signals. Fig. adapted from ref [80].

Both with and without radicals, the LLS relaxation time was significantly longer than the proton relaxation time: $\mathrm{T}_{\mathrm{LLS}}=$ $42.2 \mathrm{~s}$ at $298 \mathrm{~K}$ and $11.4 \mathrm{~T}$ without radicals and $\mathrm{T}_{\mathrm{LLS}}\left({ }^{1} \mathrm{H}\right) 16=0.6 \mathrm{~s}$ with radicals, whereas $\mathrm{T}_{1}\left({ }^{1} \mathrm{H}\right)=2.3 \mathrm{~s}$ at $298 \mathrm{~K}$ and 
7.05 $\mathrm{T}$ with radicals. Since dissolved oxygen only contributed to $\mathrm{R}_{1}=0.5 \mathrm{~s}^{-1}$ degassing the sample had no significant effect. The chemical shift difference of the two $\mathrm{H}^{\alpha}$ protons of Gly was $0.15 \mathrm{ppm}=45 \mathrm{~Hz}$ at $7.05 \mathrm{~T}$.

This experiment showed that new possibilities for the study of slow mechanisms and reactions by NMR spectroscopy become feasible. One great advantage is that the experiment only requires typical hardware and software, so that standard NMR equipment can be used. These kinds of experiments can be applied to the study of (bio)chemical reactions that are too insensitive and slow to be studied by regular NMR spectroscopy. Bodenhausen et al. demonstrated that dDNP could create long-lived polarization that could be used to obtain maps of the biodistribution of complex tracers and their metabolites. Recently, Ellis et al. presented how fumarate could be hyperpolarized via chemical reaction of acetylene dicarboxylate with para-enriched hydrogen gas leading to para-fumarate. Then, the proton singlet order in fumarate could be released as antiphase NMR signals by enzymatic conversion to malate in $\mathrm{D}_{2} \mathrm{O}$ [81].

\section{$\underline{\text { Dissolution-DNP using photo-excited triplet states }}$}

Low temperature and moderate to high magnetic fields are generally required to reach high polarizations. These conditions are usually met by using liquid helium and a superconducting magnet. This complicated and expensive instrumentation is one of the limiting factors for installing such set ups in small laboratories and hospitals. Therefore, there is a substantial need for finding a way to perform dDNP experiments at room temperatures. For instance, several groups have been trying to reach high polarization at room temperature with Overhauser DNP. But Overhauser DNP has sample volume limitations ( $\max 100 \mu \mathrm{L}$ ), and it only provides enhancements of one-to-two orders of magnitude [82, 83].

Unfortunately, when using standard radicals, the room temperature electron spins polarization is intrinsically small when working at magnetic field values typical for $\mathrm{dDNP}$ (e.g. 1.5\% at $6.7 \mathrm{~T}$ ). One way to overcome this limitation is to use photoexcited electron triplet states, which do not follow the Boltzmann distribution. Conjugated molecules such as pentacene or naphthalene can be photoexcited by laser irradiation at the appropriate wavelength. Once the conjugated molecule is in a singlet excited state, it can convert, through intersystem crossing, to a triplet state. Because of the selection rules, when a triplet state is created, it shows a polarization several times higher than Boltzmann, regardless the value of temperature and magnetic field (Fig. 5a). The technique was first described in 1980 by Bachert and co-workers [84], following the discovery of CIDNP by Bargon et al. [85] and Ward et al. [86] and has been developed by many groups in the next decades [84, 87-91].

However, the triplet state rapidly decays toward the ground state on the order of hundreds of microseconds. Therefore, the electron polarization must be transferred to nearby nuclear spin within this timescale. Even though ${ }^{1} \mathrm{H}$ polarizations higher than $50 \%$ have been regularly measured in single crystals (which are not easily dissolvable), the technique struggles in efficiently hyperpolarizing water-soluble compounds to broaden the scope of this technique [92]. With this method (i.e. dubbed triplet-DNP) Kitagawa et al. have achieved a ${ }^{1} \mathrm{H}$ spin polarization 6,100 times higher than the thermal equilibrium at room temperature and $0.38 \mathrm{~T}$, and were able to dissolve the sample with no polarization losses [93]. The hyperpolarized benzoic acid molecule was miscible with a wide range of substrates, and hyperpolarized benzoic acid had already been used as a molecular spin sensor for in vitro applications [94]. 

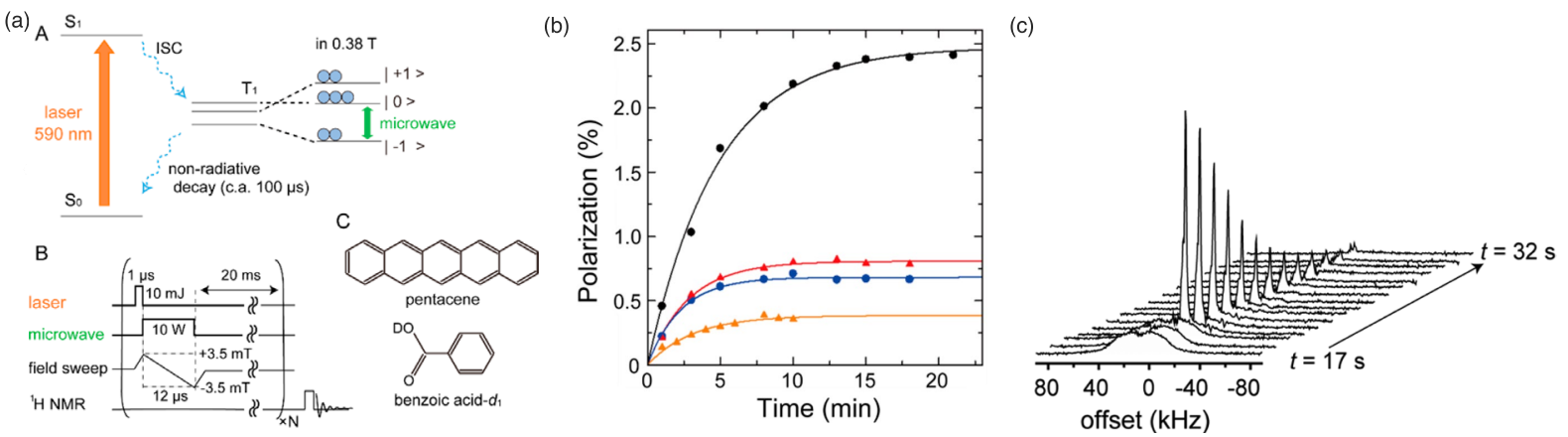

Fig. 6. (a) (A) Energy diagram of pentacene doped into benzoic acid- $d_{1}$, (B) pulse sequence for a triplet-state experiment, and (C) pentacene and benzoic acid- $d_{1}$ molecular structures. (b) ${ }^{13} \mathrm{C}$ Polarization build-ups. In all samples, the concentration of pentacene was 0.04 mol \%. (Orange and blue) powder and crystal of benzoic acid doped with pentacene- $h_{14}$, (red and black) powder and crystal of benzoic acid- $d_{1}$ doped with pentacene- $d_{14}$. (c) ${ }^{1} \mathrm{H}$ spectra recorded at $0.38 \mathrm{~T}$ and room temperature of benzoic acid- $d_{1}$ doped with pentacene- $d_{14}$ before and after dissolution. The time $\mathrm{t}=17 \mathrm{~s}$ was the time span between the end of the triplet-DNP and the beginning of the NMR measurement. Figures adapted from ref [93].

A typical sample suitable for triplet-DNP is constituted of a solid substrate, e.g. benzoic acid, doped with a small fraction of a photo-active molecule, typically pentacene or 5,12-diazatetracene. The substrate usually does not contain more than $0.05 \%$ of photo-active molecules. The sample, placed in a small magnetic field (e.g. $0.38 \mathrm{~T}$ ), is irradiated by laser light (e.g. $590 \mathrm{~nm}$ ) to create the triplet state and boost the electron polarization (Fig. 6a). The latter, as high as $25 \%$, has a lifetime of about $100 \mu \mathrm{s}$. In the meanwhile, the polarization is coherently transferred to nearby proton spins via integrated solid effect (ISE) [95, 96]: as long as microwave irradiation is applied, the magnetic field is swept adiabatically through the photo-excited radical ESR line. This procedure allows all electron spins to positively contribute to the polarization transfer to proton. By repeating the sequence (Fig. 6b) several times, the ${ }^{1} \mathrm{H}$ spin polarization of the sample is progressively increased. The polarization plateau is typically within a fraction of 1 hour.

Using deuterated precursors increases the triplet state life time, thus increasing the achievable nuclear polarization [88, 89, 93]. Usually, single crystal precursors perform better than a polycrystalline dispersion because the electron polarization and its ESR frequency dispersion both depend on the molecular orientation with respect to the external magnetic field [91, 92]. Therefore, in the latter case, the fraction of molecules having both a high electronic triplet state polarization and the right ESR frequency is smaller, resulting in only few molecules participating to the DNP process.

Negoro et al. managed for the first time to couple triplet-DNP to a dissolution experiment [93]. In Fig 5c we report the proton signal arising from benzoic acid- $d_{1}$ doped with pentacene- $d_{14}$ (red triangle in Fig. 6b) before and after dissolution. Pentacene molecules were quickly filtered prior to liquid-state NMR acquisition. No loss of polarization was observed when melting the sample to obtain a liquid-state proton polarization of $0.8 \%$ (i.e. an enhancement of 6100 compared to thermal equilibrium at $0.38 \mathrm{~T}$ ).

Polarization level and sample size are far from what can be achieved using conventional dDNP, but this new method is in its infancy and optimization has still to come. Moreover, it is worth noting that paramagnetism is active only during laser irradiation. This represents a substantial advantage when considering transporting and dissolving the sample in remote locations. This method shows that hyperpolarization at room temperature beyond the Boltzmann electron spin polarization theoretical limit is feasible, with build-up times comparable to other typical dDNP methods, and with a large range of organic solvents dissolving in benzoic acid. Next steps for triplet-DNP include hyperpolarizing larger samples, 
reaching higher polarizations in powdered samples, and hyperpolarizing water-soluble compounds to broaden the application range [97].

We note that ${ }^{13} \mathrm{C}$ spins can as well be hyperpolarized at room temperature via DNP using an optically induced electron spin polarization of diamond nitrogen vacancy centers. In 2015, Pines et al. [98] observed a bulk nuclear spin polarization of $6 \%$. This method works in principle at any non-zero magnetic field and can be used for both solids and liquids at room temperature conditions. However, the transfer of polarization from the $\mathrm{NV}$ center to ${ }^{13} \mathrm{C}$ spins within the diamond and further to nuclear spins at the surface and beyond is still being investigated.

\section{New dDNP methods in terms of hardware}

\section{Cryo-free polarizers}

As mentioned earlier, in dDNP high solid-state electron spin polarization $\left(\mathrm{P}_{\mathrm{e}}\right)$ is a key requirement to obtain a final high nuclear spin polarization after dissolution. Similarly to nuclear spins, the $\mathrm{P}_{\mathrm{e}}$ value is the result of two competing contributions: the magnetic energy, growing with the magnetic field, and the thermal energy, increasing with temperature. Fig. 7 shows $\mathrm{P}_{\mathrm{e}}$ as a function of the $\mathrm{B}_{0} / \mathrm{T}$ ratio. More than the magnetic field value, it is clear from the graph that the dominating factor is the temperature, meaning that the use of liquid helium is a key requirement for a dDNP machine.

The original dDNP setup, and its modifications across the year, have been based on a "wet" cryostat fed with an external reservoir of liquid helium [12, 99-101]. These polarizers usually consume $>2$ L of helium per sample, plus the helium needed for cooling down the system [102]. It is likely to use $100 \mathrm{~L}$ of liquid helium per week, when using a "wet" dDNP polarizer at its full capability. The latter translates into significant running costs. Moreover, it is inconvenient to frequently handle cryogenic liquids in clinical and pre-clinical environments.

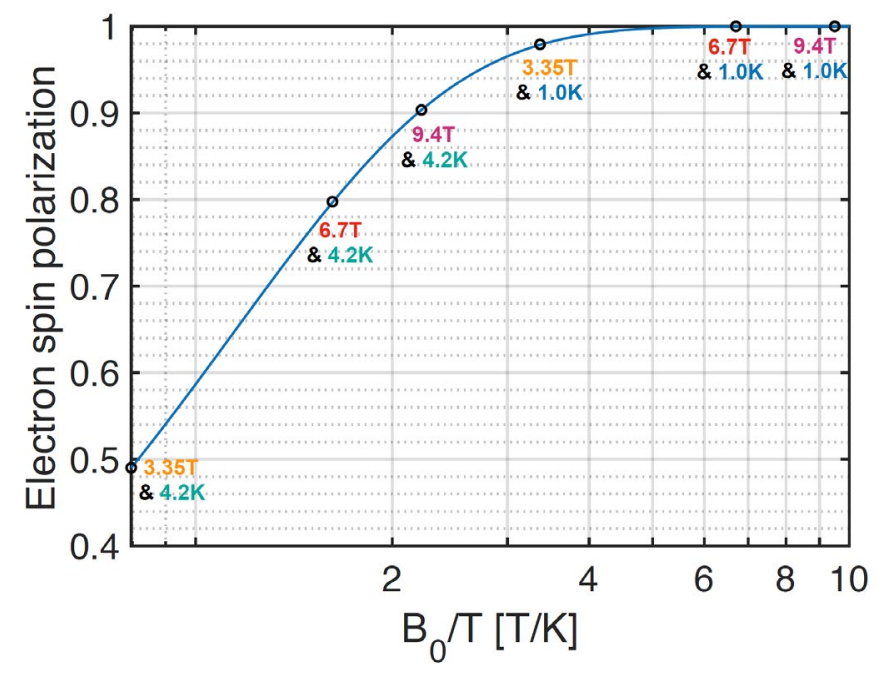

Fig. 7. Electron spin polarization as a function of the ratio $\mathrm{B}_{0} / \mathrm{T}$.

To this hand, the need for "cryogen free" polarizers became more and more stringent across the years. The SPINlab ${ }^{\mathrm{TM}}$ " polarizer designed and commercialize by General Electric (Boston, Massachusetts, United States) was the first "cryogen 
free polarized", and was meant for sterile use [38]. Differently from a "wet" polarizer, it is equipped with a cryo-cooler and a sorption pump able to condense helium gas inside the sample space for a full working day. The SPINlab ${ }^{\mathrm{TM}}$, operating at $\mathrm{B}_{0}=5 \mathrm{~T}$ and $\mathrm{T}=0.8 \mathrm{~K}$, is a sophisticated system that fulfills the strict requirements of a clinical environment. However, the goals for research and pre-clinical use are different and the hardware can be somewhat simplified.

The first "cryogen free" polarizer for pre-clinical use was introduced by Ardenkjaer-Larsen in 2017 at the ENC conference $[103,104]$. The polarizer is based on a dry magnet that is cooled by an integrated cryo-cooler. Once the polarizer reaches a temperature of $\mathrm{T}=2.5 \mathrm{~K}$, the magnetic field is progressively increased in approximately one hour, and the inlet port of the cryostat is connected to a storage tank containing helium gas. Helium is then condensed through a charcoal trap, and the liquid helium is then delivered through a needle valve into the VTI containing the DNP probe. A dry pump reduces the VTI pressure, and the evaporated helium travels back to the gas storage tank to be re-liquefied into the helium pot in a continuous cycle. The described cycle is shown in Fig. 8a [105]. A temperature of $\mathrm{T}=1.49 \mathrm{~K}$ can be attained as a base temperature within 100 to $180 \mathrm{~min}$.
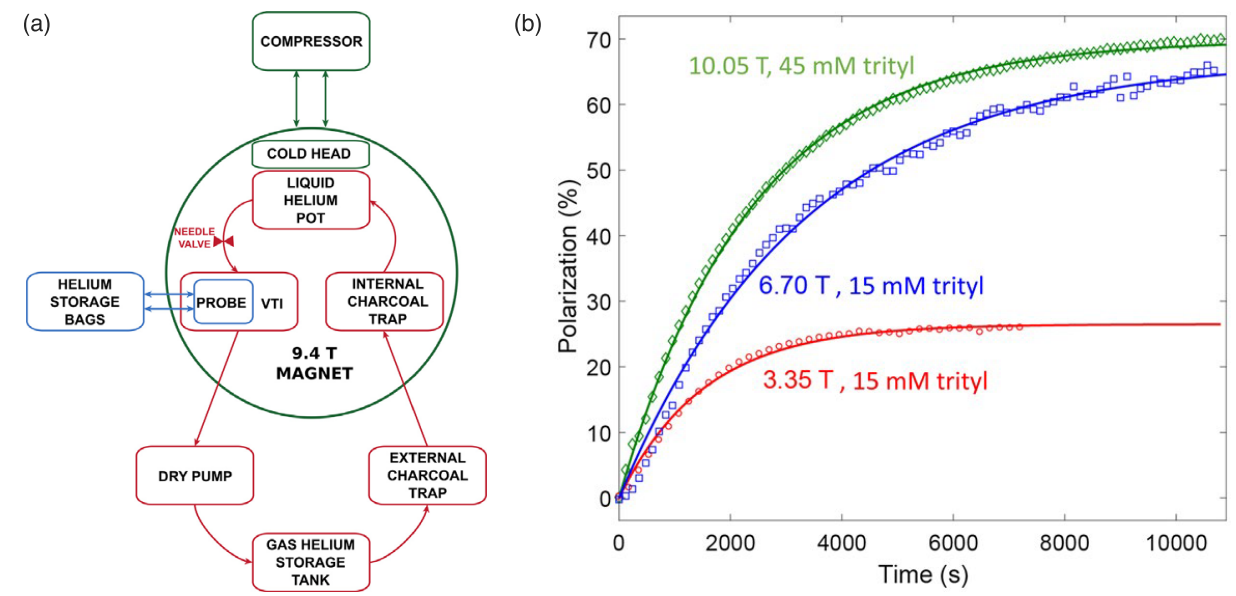

Fig. 8. (a) Schematic of the three helium gas circuits used in the apparatus. (b) DNP build-up curves at $3.35 \mathrm{~T}$ (red circles, $15 \mathrm{mM}$ of AH111501, 93.88 $\mathrm{GHz}$, and $40 \mathrm{~mW}$ of microwave irradiation), $6.7 \mathrm{~T}$ (blue squares, $15 \mathrm{mM}$ of AH111501, $187.46 \mathrm{GHz}, 20 \mathrm{MHz}$ modulation at $1 \mathrm{kHz}$, and $30 \mathrm{~mW}$ microwave of irradiation), and $10.1 \mathrm{~T}$ (green diamonds, $45 \mathrm{mM}$ of AH111501, $281.87 \mathrm{GHz}, 40 \mathrm{MHz}$ modulation at $1 \mathrm{kHz}$, and $18 \mathrm{~mW}$ of microwave irradiation) of [1- $\left.{ }^{13} \mathrm{C}\right]$ pyruvic acid at $1.3 \mathrm{~K}$. (a) was taken from ref [49] and (b) was taken from ref [105].

Two research groups: Ardenkjær-Larsen et al. and Jannin et al. published the same year their ${ }^{13} \mathrm{C}$ polarization results using a cryogenic-free polarizer built on the same dry magnet provided by Cryogenic Ltd (Unit 6 The Vale, Acton, London W3 7QE, United Kingdom) [49, 105]. In Fig. 8b are shown DNP build-up curves at 94, 188, and 282 GHz of [1${ }^{13} \mathrm{C}$ ]pyruvic acid doped with trityl AH111501. Even though the field could be continuously ramped to $10 \mathrm{~T}$, only discrete field values were explored depending on the availability of microwave sources. Microwave sources suitable for DNP are widely available at $94 \mathrm{GHz}$. The frequency can then be multiplied by a doubler or tripler to match the electron Larmor frequency at the desired field. To obtain optimal DNP performance, the radical concentration was modified according to the magnetic field value $(15 \mathrm{mM}$ at $3.35 \mathrm{~T}, 30 \mathrm{mM}$ at $6.70 \mathrm{~T}, 45 \mathrm{mM}$ at $10.10 \mathrm{~T})$. At higher magnetic field, higher radical concentration was required. Differently, keeping the radical concentration to $15 \mathrm{mM}$, microwave frequency modulation was beneficial to improve the enhancement at $6.70 \mathrm{~T}$ and $10.10 \mathrm{~T}$. At optimal conditions, no improvement in polarization 
was observed between $6.70 \mathrm{~T}$ and $10.10 \mathrm{~T}$ on $\left[1-{ }^{13} \mathrm{C}\right]$ pyruvic acid. The maximum achievable ${ }^{13} \mathrm{C}$ polarization was of 70 $\%$ in both cases, but the time need increased from 1 to $3 \mathrm{~h}$ by increasing the field from $6.70 \mathrm{~T}$ to $10.10 \mathrm{~T}$.

While, Ardenkjaer-Larsen et al. used the fluid path technology to dissolve the samples [38], Jannin et al. used a classical dissolution wand. This choice led to important polarization losses. Indeed, the dissolution wand introduces in the system a considerable amount of heat that might cause the quench of the dry magnet, unless the dissolution is performed at sufficient distance from the isocenter, thus at low magnetic field. What characterized Jannin et al. work was the implementation of ${ }^{1} \mathrm{H}$-to- ${ }^{13} \mathrm{C}$ cross polarization in a cryogen-free magnet. Jannin et al. hyperpolarized a sample composed of $80 \mathrm{mM}$ of TEMPOL, which was the optimal radical molecule to polarize ${ }^{1} \mathrm{H}$, in a mixture glycerol- $d_{8}: \mathrm{D}_{2} \mathrm{O}: \mathrm{H}_{2} \mathrm{O}$ (60:30:10), with $1.5 \mathrm{M}$ of $\left[1-{ }^{13} \mathrm{C}\right]$ sodium acetate [105]. At $9.4 \mathrm{~T}$, multiple $\mathrm{CP}$ contacts allowed to reach a ${ }^{13} \mathrm{C}$ polarization of $50 \%$ in about $50 \mathrm{~min}$. Each CP step was performed every $5.3 \mathrm{~min}$, in order to restore the ${ }^{1} \mathrm{H}$ polarization via direct DNP. The CP efficiency was maximized by applying microwave gating [106] during the spin lock to prevent electron spins induced ${ }^{1} \mathrm{H}$ nuclei fast relaxation.
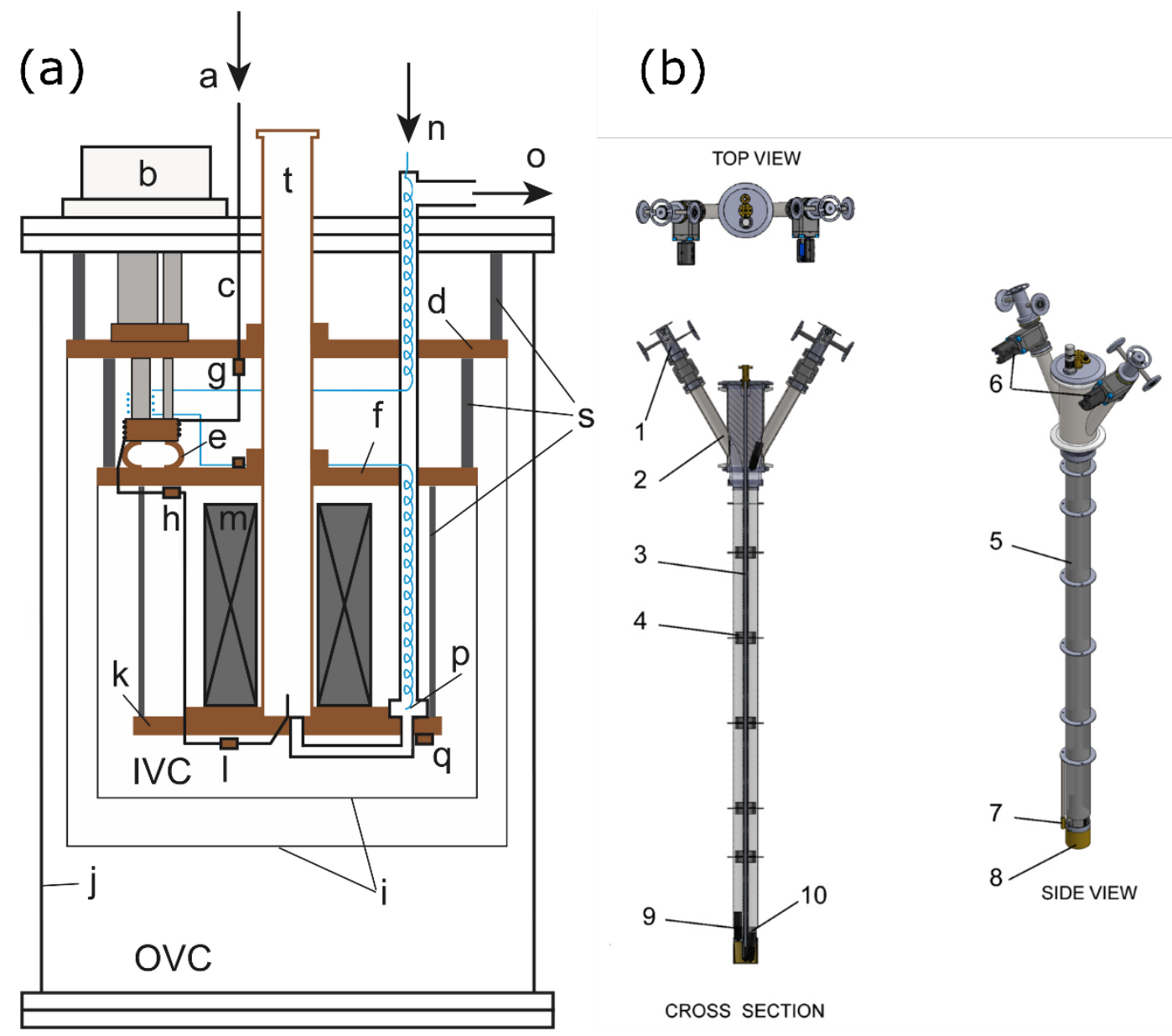

CROSS SECTION

Fig. 9. Schematic drawing of the cryostat without insert: (a) helium gas inlet, (b) cryocooler, (c) helium-filling capillary, (d) 50-K plate, (e) copper flexible strap, (f) 3-K plate, (g) thermometer on 50-K plate, (h) thermometer on 3-K plate, (i) radiation shields, (j) vacuum can, (k) 1-K plate, (l) RuO2 thermometer on helium inlet, (m) magnet, (n) input port of 1-K recirculation circuit to high-impedance capillary, (o) output port of 1-K recirculation circuit to dry vacuum pump, (p) 1-K pot, (q) RuO2 thermometer on 1-K plate, (s) supporting rods and (t) sample tube. The figure was from ref [50].

More recently, a third version of "cryogen free" dDNP polarizer was introduced by Comment et al. [50]. Here, the geometry (see Fig. 9a) allowed more flexibility as regarding sample space operations. In particular, the physical separation between the sample tube $(\mathrm{t})$ and $1 \mathrm{~K}$ pot $(\mathrm{p})$ lifts the need to have a DNP probe that is leak tight. Moreover, this work was 
the first to show a multi-sample probe for pre-clinical use on a cryogen-free cryostat (see Fig 9b). Previously, Ernst et al. presented a multi-sample DNP probe (i.e. the revolver) for a traditional wet cryostat [107].

\section{Fluid path}

The fluid path in its original version is a system introduced for the first time in 2011 for sterile use [38]. More recently, driven by the need to make this technology compatible with dDNP UV-samples, A. Capozzi et al. developed a modified device that is reusable and suitable from solid/frozen samples loading [20, 75, 108]. The custom fluid path (CFP), reported in Fig. 10, does not present any glued joint in order to improve durability and success rate during dissolutions. The main novelty consists in a home-made threaded sample vial (Fig. 10e). The latter, able to contain a volume up to $0.4 \mathrm{~mL}$, is made of natural polyether ether ketone (PEEK) plastic and divided in two parts that can screwed together: the neck and the body. The vial neck is laser welded to a black PEEK outer lumen. Compressing a PTFE O-ring (white circles in Fig. 10e) between the vial neck and body makes the system leak-tight to superfluid helium, a key feature to perform the dissolution step [75]. The inner lumen ends with a press fit PEEK nozzle (green part in Fig. 10e) allowing the hot solvent to be sprayed as a mist on the sample for a more efficient dissolution. The dynamic sealing (Fig. 10d) includes two double lip seals: two PTFE lip seals (white circles in Fig. 10d) are placed against the outer lumen and two rubber lip seals (black circles in Fig. 10d) are pushing against the airlock chamber. Both O-rings are chosen to minimize resistance and have a low dynamic leak rate (as good as $10^{-7} \mathrm{mbar} \cdot 1 / \mathrm{s}$ ), providing efficient sealing between the inside and outside of the cold VTI. The sample vial is first inserted into the airlock, and the dynamic sealing pushed onto the airlock. Then, the chamber is flushed with helium gas to remove any moisture trace, and the gate valve is then opened for the sample to be lowered down to the probe coil. A "tee" valve (Fig. 10c) splits the paths of the arriving hot solvent and the leaving hyperpolarized liquid. A home-made shrinking adapter (green part in Fig. 10c) makes the transition between the outer and inner lumen. The top of the fluid path ends with a "quick-release" connector (Fig. 10a \& b) allowing the fluid path to be conveniently moved from the leak test station to the polarizer. The dissolution head is composed of a home-made boiler, ringed with heating stripes, allowing the solvent to be warmed up at constant volume. A manometer is used to monitor the pressure evolution during heating, and a one-way valve is added to prevent any hot solvent from reaching the helium gas bottle (more details on the dissolution head can be found in ref [20]). Once the solvent container has reached the desired pressure, the sample vial is lifted above the liquid helium level inside the VTI, the quick release connected to the dissolution head, and the hot solvent quickly injected through the inner lumen of the fluid path. The sample melts immediately and is blown off running first inside the volume between the outer and inner lumen, and finally inside the horizontal harm of the "tee" (right black part in Fig. 10c).

(a)

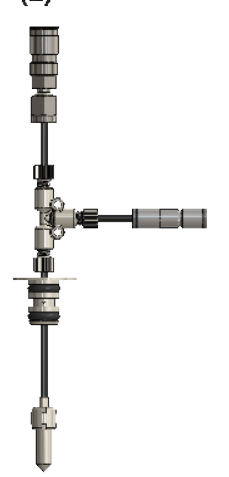

(b)

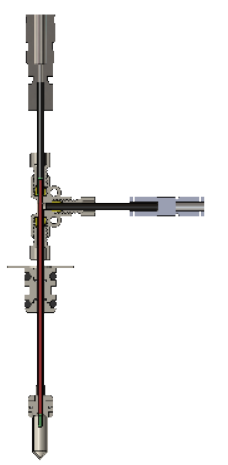

(c)

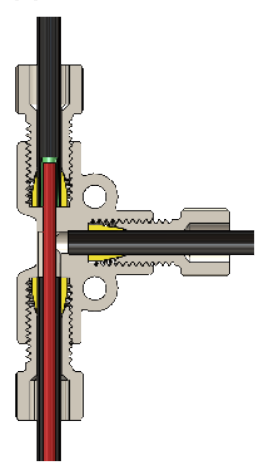

(d)

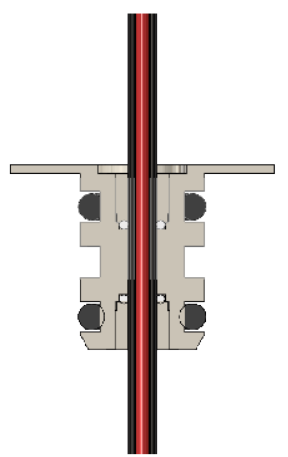

(e)

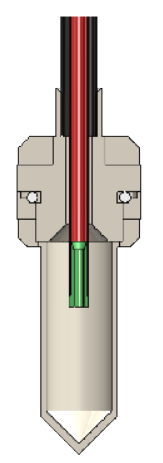


Fig. 10. (a) Entire custom fluid-path view. (b) Entire custom fluid-path section. (c) Zoom on the "tee" valve section. (d) Zoom on the dynamic sealing section. (e) Zoom on the sample cup section.

Using a fluid path dissolution system, has the big advantage to run the VTI constantly at low pressure (typically 1 mbar), minimizing the heat load introduced by the polarizer pressurization by the insertion of a traditional dissolution wand. This is particularly useful when working with a system with limited cooling power such as a "cryogen free" polarizer.

\section{Bullet DNP}

The dissolution step leads to a dilution of the solid-state sample depending on the system and specific experiment. Unfortunately, the dissolution buffer volume cannot be decreased as lib because a certain minimum heat capacity is necessary to avoid freezing of the liquid during the dissolution step while travelling towards the cold part of the VTI. This can represent a drawback for in vitro and chemical applications were the solid-state sample is usually small and a dilution factor of 100-fold is typically obtained. It is possible to reach higher target concentrations by co-dissolving an immiscible phase to the dissolution buffer. However, when 5-mm NMR tubes are used, the separation between the two solvents is incomplete, which leads to a broadening of the lines up to hundreds of $\mathrm{Hz}$ [22]. Moreover, transferring the HP sample from the polarizer to the measuring apparatus as a liquid cannot be faster than 1-2 s, even for optimized pushing systems employing pressurized lines [109]. This time interval can be non-negligible on the substrate $\mathrm{T}_{1}$ scale, especially when working in presence of paramagnetic impurities and/or larger molecules.

An alternative is what is known as "bullet-DNP" [110]. In 2019, taking inspiration from a non-DNP setup developed by Bruker in the context of transport of hyperpolarization achieved via "brute force" [2], Meier et al. showed that a liquidstate ${ }^{13} \mathrm{C}$ polarization of $>30 \%$ could be obtained for a $\left[1-{ }^{13} \mathrm{C}\right] \mathrm{PA}$ sample doped with trityl by dissolving it directly inside the measuring apparatus in a small volume. This was possible provided that the distance between the polarizer and measuring apparatus was covered in a short time $(<100 \mathrm{~ms})$, and the path "sheltered" by an appropriate magnetic field [110]. Since the hot buffer does not travel through the DNP polarizer, a dilution volume as small as $700 \mu \mathrm{L}$ could be used to melt a $80 \mu \mathrm{L}$ solid sample, reducing the dilution factor to approx. 10-fold.
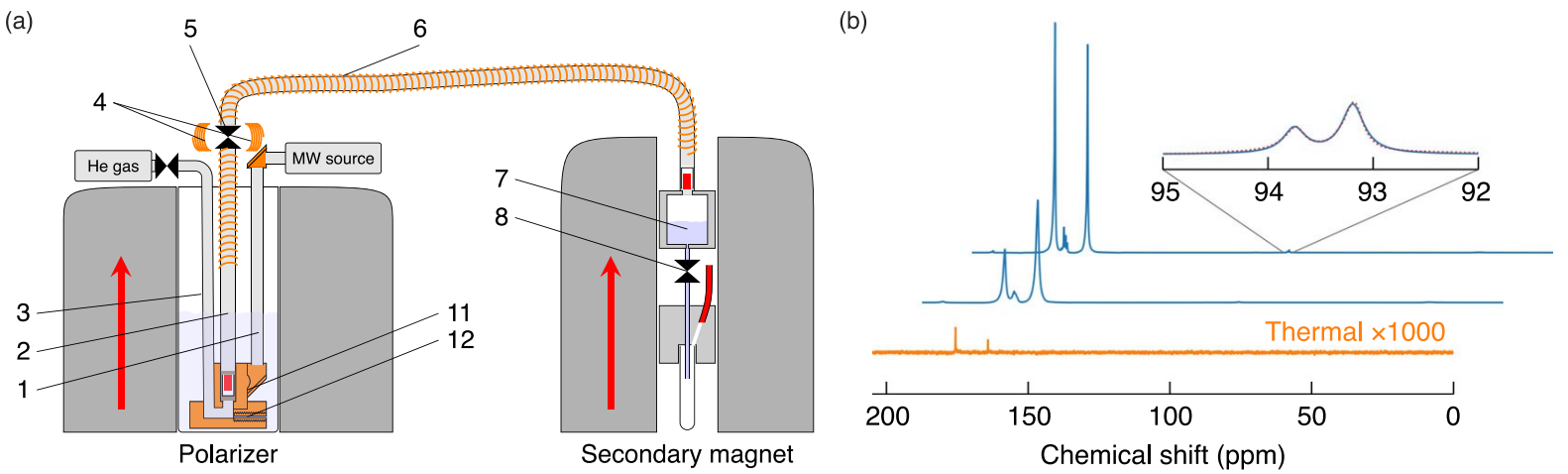

Fig. 11. (a) Schematic drawing of the bullet-DNP setup. It includes a polarizer, a tube for transfer (6) enveloped with a solenoid, and an injector. The red arrows indicate the orientation of both magnets. The valve (5) allows a change in the magnetic field direction with two Helmholtz coils (4). (3) Tube blowing helium gas, (2) main tube and (1) microwave pipe. (11) Brass piece for tuning and matching. (12) Brass piece sealing the transfer path. More details can be found in ref [110] (b) ${ }^{13} \mathrm{C}$ DNP enhanced NMR spectra of hyperpolarized $1-{ }^{13} \mathrm{C}$-pyruvic acid. Fig. taken from ref $[110]$. 
In Fig. 11a we report the experimental setup. Being the sample doped with permanent radicals, the presence of a magnetic field changing direction and value adiabatically with respect to the trajectory of the bullet was a key requirement to preserve the hyperpolarization during transfer. This magnetic field was obtained by enveloping the transfer tube with a solenoid and feeding it a current pulse of 60 A for the duration of the transfer. A magnetic field higher than $75 \mathrm{mT}$ along the all transfer path was obtained. This value was chosen to avoid any low field thermal mixing between the highly polarized ${ }^{13} \mathrm{C}$ nuclear spins and the fast relaxing and poorly polarized proton spins $[111,112]$.

In Fig. $11 \mathrm{~b}$ we report the ${ }^{13} \mathrm{C}$ liquid-state NMR signal after dissolution in $\mathrm{D}_{2} 0$. If this method has the big advantage of considerably reducing the dissolution volume while preserving high polarization, the $30 \mathrm{~Hz}$ line broadening, coming from small helium bubbles generated by the impact of the bullet with the hot solvent, has to be improved if this new technique targets high-resolution NMR applications.

\section{Discussion and conclusion}

Each new technological and methodological advance in dissolution-DNP has its merits and challenges and serves specific purposes. The requirements are quite different depending on whether the target is clinical imaging, pre-clinical imaging or in vitro NMR. In most cases the highest possible polarization is indispensable, since dDNP is a single-shot-technique (no averaging). However, throughput is also a consideration that can have several solutions: multiple samples, faster polarization (e.g. CP), efficient sample loading and unloading, or batch polarization (storage of polarized samples for remote dissolution). For all applications, reproducibility and reliability are essential for analysis, modelling and quantification. Therefore, the hyperpolarization experiment not only involves the actual hyperpolarization, but equally the fast and efficient detection of the nuclear magnetizations. These two topics are beyond the scope of this review, but fast transfer/injection and efficient acquisition strategies should receive equal attention.

Dissolution-DNP is a developing research field. Even though polarization close to unity has already been reached, the limits are constantly being pushed with new innovative methods and instrumentation. The samples' biocompatibility, transport of hyperpolarized substrates to remote location, the required high field and low temperatures, high helium costs, high dilution factors and relaxation are all limitations that are currently being solved by smart scientists. The recent technical advances are now paving the way for making dissolution-DNP a method of choice for hyperpolarized magnetic resonance, and hyperpolarization a method of choice for NMR and MRI.

\section{Compliance with Ethical Standards}

JA is an employee of GE Healthcare and the owner of the company Polarize. We have no further potential conflicts of interest. This review does not involve any human participants or animal research.

\section{Authors' Contributions}

JA review article conception; ACP drafting of manuscript; AC and JA critical revision.

\section{References}

1. Abragam A (1961) The principles of nuclear magnetism. University Press, Oxford 
2. Hirsch ML, Kalechofsky N, Belzer A, Rosay M, Kempf JG (2015) Brute-Force Hyperpolarization for NMR and MRI. J Am Chem Soc 137:8428-8434.

3. Walker TG, Happer W (Apr) Spin-exchange optical pumping of noble-gas nuclei. Rev Mod Phys 69:629-642.

4. Natterer J, Bargon J (1997) Parahydrogen induced polarization. Prog Nucl Magn Reson Spectrosc 31:293-315.

5. Abragam A, Goldman M (1978) Principles of Dynamic Nuclear-Polarization. Rep Prog Phys 41:395-467.

6. Overhauser AW (1953) Polarization of nuclei in metals. Phys Rev 92:411.

7. Carver TR, Slichter CP (1953) Polarization of nuclear spins in metals. Phys Rev 92:212.

8. van den Brandt B, Hautle P, Konter JA, Kurdzesau F (2008) Dynamic Nuclear Polarization - from Polarized Targets to Metabolic Imaging. Appl Magn Reson 34:475-481.

9. Becerra LR, Gerfen GJ, Temkin RJ, Singel DJ, Griffin RG (1993) Dynamic nuclear polarization with a cyclotron resonance maser at $5 \mathrm{~T}$. Phys Rev Lett 71:3561-3564.

10. Gerfen GJ, Becerra LR, Hall DA, Griffin RG, Temkin RJ, Singel DJ (1995) High frequency (140 GHz) dynamic nuclear polarization: polarization transfer to a solute in frozen aqueous solution. J Chem Phys 102:9494-9497.

11. Griesinger C, Bennati M, Vieth HM, Luchinat C, Parigi G, Höfer P, Engelke F, Glaser SJ, Denysenkov V, Prisner TF (2012) Dynamic nuclear polarization at high magnetic fields in liquids. Prog Nucl Magn Reson Spectrosc 64:4 28.

12. Ardenkjaer-Larsen JH, Fridlund B, Gram A, Hansson G, Hansson L, Lerche MH, Servin R, Thaning M, Golman K (Sep 2) Increase in signal-to-noise ratio of $>10,000$ times in liquid-state NMR. Proc Natl Acad Sci U S A 100:10158-10163.

13. Ardenkjaer-Larsen JH (2016) On the present and future of dissolution-DNP. J Magn Reson 264:3-12.

14. Nelson SJ, Kurhanewicz J, Vigneron DB, Larson PEZ, Harzstark AL, Ferrone M, van Criekinge M, Chang JW, Bok R, Park I, Reed G, Carvajal L, Small EJ, Munster P, Weinberg VK, Ardenkjaer-Larsen JH, Chen AP, Hurd RE, Odegardstuen LI, Robb FJ, Tropp J, Murray JA (Aug 14) Metabolic Imaging of Patients with Prostate Cancer Using Hyperpolarized [1- ${ }^{13 \$}$ C]Pyruvate. Sci Transl Med 5:198ra108 1-10.

15. van Heeswijk RB, Uffmann K, Comment A, Kurdzesau F, Perazzolo C, Cudalbu C, Jannin S, Konter JA, Hautle P, van den Brandt B, Navon G, van der Klink JJ, Gruetter R (Jun) Hyperpolarized ${ }^{6 \$} \mathrm{Li}$ as a Sensor of Nanomolar Contrast Agents. Magn Reson Med 61:1489-1493.

16. Bowen S, Hilty C (2008) Time-Resolved Dynamic Nuclear Polarization Enhanced NMR Spectroscopy. Angew Chem 120:5313-5315.

17. Lee Y, Heo GS, Zeng H, Wooley KL, Hilty C (2013) Detection of living anionic species in polymerization reactions using hyperpolarized NMR. J Am Chem Soc 135:4636-4639.

18. Lerche MH, Jensen PR, Karlsson M, Meier S (2015) NMR insights into the inner workings of living cells. Anal Chem 87:119-132.

19. Harris T, Eliyahu G, Frydman L, Degani H (2009) Kinetics of hyperpolarized C-13(1)-pyruvate transport and metabolism in living human breast cancer cells. Proc Natl Acad Sci U S A 106:18131-18136.

20. Pinon AC, Capozzi A, Ardenkjær-Larsen JH (2020) Hyperpolarized water through dissolution dynamic nuclear polarization with UV-generated radicals. Commun Chem 3:57.

21. Olsen G, Markhasin E, Szekely O, Bretschneider C, Frydman L (2016) Optimizing water hyperpolarization and dissolution for sensitivity-enhanced 2D biomolecular NMR. J Magn Reson 264:49-58.

22. Szekely O, Olsen GL, Felli IC, Frydman L (2018) High-Resolution 2D NMR of Disordered Proteins Enhanced by Hyperpolarized Water. Anal Chem 90:6169-6177. 
23. Harris T, Szekely O, Frydman L (2014) On the Potential of Hyperpolarized Water in Biomolecular NMR Studies. J Phys Chem B 118:3281-3290.

24. Chappuis Q, Milani J, Vuichoud B, Bornet A, Gossert AD, Bodenhausen G, Jannin S (2015) Hyperpolarized Water to Study Protein-Ligand Interactions. J Phys Chem Lett 6:1674-1678.

25. Wenckebah WTh (2016) Essentials of Dynamic Nuclear Polarization. Ridderprint B.V. Ridderkerk, The Netherlands

26. de Boer W (1976) Dynamic orientation of nuclei at low temperatures. J Low Temp Phys 22:185-212.

27. de Boer W, Borghini M, Morimoto K, Niinikoski TO, Udo F (1974) Dynamic polarization of protons, deuterons, and carbon-13 nuclei: Thermal contact between nuclear spins and an electron spin-spin interaction reservoir. J Low Temp Phys 15:249-267.

28. Wind RA, Duijvestijn MJ, van der Lugt C, Manenschijn A, Vriend J (1985) Applications of dynamic nuclear polarization in ${ }^{13 \$} \mathrm{C}$ NMR in solids. Prog Nucl Magn Reson Spectrosc 17:33-67.

29. Borghini M (1971) Mechanism of nuclear dynamic polarization by electron-nucleus dipolar coupling in solids. European Organization for Nuclear Research, Geneva (Switzerland)

30. Borghini M (1968) Spin-Temperature Model of Nuclear Dynamic Polarization Using Free Radicals. Phys Rev Lett 20:419-.

31. Hwang CF, Hill DA (1967) Phenomenological Model for the New Effect in Dynamic Polarization. Phys Rev Lett 19:1011-1014.

32. Guarin D, Marhabaie S, Rosso A, Abergel D, Bodenhausen G, Ivanov KL, Kurzbach D (2017) Characterizing Thermal Mixing Dynamic Nuclear Polarization via Cross-Talk between Spin Reservoirs. J Phys Chem Lett 8:5531-5536.

33. Hurd RE, Yen Y-F, Chen A, Ardenkjaer-Larsen J-H (2012) Hyperpolarized ${ }^{13 \$} \mathrm{C}$ metabolic imaging using dissolution dynamic nuclear polarization. J Magn Reson Imaging 36:1314-1328.

34. Heckmann J, Meyer W, Radtke E, Reicherz G (Oct) Electron spin resonance and its implication on the maximum nuclear polarization of deuterated solid target materials. Phys Rev B 74:134418(1-9).

35. Johanneson H, Macholl S, Ardenkjaer-Larsen JH (Apr) Dynamic Nuclear Polarization of [1-13\$C]pyruvic acid at 4.6 T. J Magn Reson 197:167-175.

36. Cheng T, Capozzi A, Takado Y, Balzan R, Comment A (2013) Over 35\% liquid-state ${ }^{13 \$}$ C polarization obtained via dissolution dynamic nuclear polarization at $7 \mathrm{~T}$ and $1 \mathrm{~K}$ using ubiquitous nitroxyl radicals. Phys Chem Chem Phys 15:20819-20822.

37. Jannin S, Bornet A, Melzi R, Bodenhausen G (2012) High field dynamic nuclear polarization at $6.7 \mathrm{~T}$ : ${ }^{13 \$} \mathrm{C}$ polarization above 70\% within 20 min. Chem Phys Lett 549:99-102.

38. Ardenkjaer-Larsen J-H, Leach AM, Clarke N, Urbahn J, Anderson D, Skloss TW (2011) Dynamic nuclear polarization polarizer for sterile use intent. NMR Biomed 24:927-932.

39. Comment A, Merritt ME (Dec 2) Hyperpolarized Magnetic Resonance as a Sensitive Detector of Metabolic Function. Biochemistry 53:7333-7357.

40. Capozzi A, Patel S, Wenckebach WT, Karlsson M, Lerche MH, Ardenkjær-Larsen JH (2019) Gadolinium Effect at High-Magnetic-Field DNP: $70 \%{ }^{13}$ C Polarization of [U- ${ }^{13}$ C] Glucose Using Trityl. J Phys Chem Lett 10:34203425 .

41. Friesen-Waldner L, Chen A, Mander W, Scholl TJ, McKenzie CA (2012) Optimisation of dynamic nuclear polarisation of [1-(13)C] pyruvate by addition of gadolinium-based contrast agents. J Magn Reson 223:85-9.

42. Lumata L, Merritt ME, Malloy CR, Sherry AD, Kovacs Z (2012) Impact of Gd3+ on DNP of [1- ${ }^{13 \$}$ C] pyruvate doped with trityl OX063, BDPA, or 4-oxo-TEMPO. J Phys Chem A 116:5129-5138. 
43. Bornet A, Milani J, Vuichoud B, Linde AJP, Bodenhausen G, Jannin S (2014) Microwave frequency modulation to enhance Dissolution Dynamic Nuclear Polarization. Chem Phys Lett 602:63-67.

44. Kiselev YF, Niinikoski TO (1996) EPR and dynamic nuclear polarization with frequency modulation. Amsterdam, The Netherlands, pp 389-391

45. Adeva B, al. (1996) Large enhancement of deuteron polarization with frequency modulated microwaves. Nucl Instrum Methods Phys Res Sect -Accel Spectrometers Detect Assoc Equip 372:339-343.

46. Bornet A, Melzi R, Linde AJP, Hautle P, van den Brandt B, Jannin S, Bodenhausen G (Jan 3) Boosting Dissolution Dynamic Nuclear Polarization by Cross Polarization. J Phys Chem Lett 4:111-114.

47. Kurdzesau F, van den Brandt B, Comment A, Hautle P, Jannin S, van der Klink JJ, Konter JA (Aug 7) Dynamic nuclear polarization of small labelled molecules in frozen water-alcohol solutions. J Phys -Appl Phys 41:155506 1-10.

48. Milani J, Vuichoud B, Bornet A, Miéville P, Mottier R, Jannin S, Bodenhausen G (2015) A magnetic tunnel to shelter hyperpolarized fluids. Rev Sci Instrum 86:024101.

49. Ardenkjær-Larsen JH, Bowen S, Petersen JR, Rybalko O, Vinding MS, Ullisch M, Nielsen NC (2018) Cryogen-Free dissolution Dynamic Nuclear Polarization polarizer operating at 3.35 T, 6.70 T and 10.1 T. Magn. Reson. Med. accepted:

50. Cheng T, Gaunt AP, Marco-Rius I, Gehrung M, Chen AP, Klink JJ, Comment A (2020) A multisample 7 T dynamic nuclear polarization polarizer for preclinical hyperpolarized MR. NMR Biomed. doi: 10.1002/nbm.4264

51. Yoshihara HAI, Can E, Karlsson M, Lerche MH, Schwitter J, Comment A (2016) High-field dissolution dynamic nuclear polarization of [1-C-13]pyruvic acid. Phys Chem Chem Phys 18:12409-12413.

52. Lama B, Collins JHP, Downes D, Smith AN, Long JR (2016) Expeditious dissolution dynamic nuclear polarization without glassing agents: Expeditious Dissolution DNP Without Glassing Agents. NMR Biomed 29:226-231.

53. Stephenson JL (1956) ICE CRYSTAL GROWTH DURING THE RAPID FREEZING OF TISSUES. J Biophys Biochem Cytol 2:45-52.

54. Hall RS, Board SJ, Clare AJ, Duffey RB, Playle TS, Poole DH (1969) Inverse Leidenfrost Phenomenon. Nature 224:266-267.

55. Cavagna A (2009) Supercooled liquids for pedestrians. Phys Rep 476:51-124.

56. Haida O, Suga H, Seki S (1977) Calorimetric study of the glassy state XII. Plural glass-transition phenomena of ethanol. J Chem Thermodyn 9:1133-1148.

57. Lumata L, Merritt ME, Kovacs Z (2013) Influence of deuteration in the glassing matrix on ${ }^{13 \$} \mathrm{C}$ dynamic nuclear polarization. Phys Chem Chem Phys 15:7032-7035.

58. Lumata L, Kovacs Z, Sherry AD, Malloy C, Hill S, van Tol J, Yu L, Song L, Merritt ME (2013) Electron spin resonance studies of trityl OX063 at a concentration optimal for DNP. Phys Chem Chem Phys 15:9800-9807.

59. Miéville P, Ahuja P, Sarkar R, Jannin S, Vasos PR, Gerber-Lemaire S, Mishkovsky M, Comment A, Gruetter R, Ouari O, others (2010) Scavenging free radicals to preserve enhancement and extend relaxation times in NMR using dynamic nuclear polarization. Angew Chem 122:6318-6321.

60. Cheng T, Mishkovsky M, Junk MJN, Münnemann K, Comment A (2016) Producing Radical-Free Hyperpolarized Perfusion Agents for In Vivo Magnetic Resonance Using Spin-Labeled Thermoresponsive Hydrogel. Macromol Rapid Commun 37:1074-1078.

61. Vuichoud B, Bornet A, de Nanteuil F, Milani J, Canet E, Ji X, Mieville P, Weber E, Kurzbach D, Flamm A, Konrat R, Gossert AD, Jannin S, Bodenhausen G (2016) Filterable Agents for Hyperpolarization of Water, Metabolites, and Proteins. Chem- Eur J 22:14696-14700. 
62. Gajan D, Bornet A, Vuichoud B, Milani J, Melzi R, van Kalkeren HA, Veyre L, Thieuleux C, Conley MP, Gruning WR, Schwarzwalder M, Lesage A, Coperet C, Bodenhausen G, Emsley L, Jannin S (2014) Hybrid polarizing solids for pure hyperpolarized liquids through dissolution dynamic nuclear polarization. Proc Natl Acad Sci 111:14693-14697.

63. Ji X, Bornet A, Vuichoud B, Milani J, Gajan D, Rossini AJ, Emsley L, Bodenhausen G, Jannin S (2017) Transportable hyperpolarized metabolites. Nat Commun. doi: 10.1038/Ncomms13975

64. Capozzi A, Cheng T, Boero G, Roussel C, Comment A (2017) Thermal annihilation of photo-induced radicals following dynamic nuclear polarization to produce transportable frozen hyperpolarized 13C-substrates. Nat Commun 8:15757.

65. Eichhorn TR, Takado Y, Salameh N, Capozzi A, Cheng T, Hyacinthe JN, Mishkovsky M, Roussel C, Comment A (Nov 5) Hyperpolarization without persistent radicals for in vivo real-time metabolic imaging. Proc Natl Acad Sci U S A 110:18064-18069.

66. van der Wel PCA, Hu K-N, Lewandowski J, Griffin RG (2006) Dynamic Nuclear Polarization of Amyloidogenic Peptide Nanocrystals: GNNQQNY, a Core Segment of the Yeast Prion Protein Sup35p. J Am Chem Soc 128:10840-10846.

67. Rossini AJ, Zagdoun A, Hegner F, Schwarzwälder M, Gajan D, Copéret C, Lesage A, Emsley L (2012) Dynamic Nuclear Polarization NMR Spectroscopy of Microcrystalline Solids. J Am Chem Soc 134:16899-16908.

68. Pinon AC, Schlagnitweit J, Berruyer P, Rossini AJ, Lelli M, Socie E, Tang M, Pham T, Lesage A, Schantz S, Emsley L (2017) Measuring Nano- to Microstructures from Relayed Dynamic Nuclear Polarization NMR. J Phys Chem C 121:15993-16005.

69. Pinon AC (2018) Spin Diffusion in Dynamic Nuclear Polarization Nuclear Magnetic Resonance. doi: 10.5075/EPFLTHESIS-8519

70. Pines A, Gibby MG, Waugh JS (1972) Proton-enhanced nuclear induction spectroscopy ${ }^{13 \$} \mathrm{C}$ chemical shielding anisotropy in some organic solids. Chem Phys Lett 15:373-376.

71. Suter D, Ernst RR (1985) Spin diffusion in resolved solid-state NMR spectra. Phys Rev B 32:5608-5627.

72. Szeverenyi NM, Sullivan MJ, Maciel GE (1982) Observation of spin exchange by two-dimensional fourier transform 13C cross polarization-magic-angle spinning. J Magn Reson 1969 47:462-475.

73. Silverio DL, van Kalkeren HA, Ong T-C, Baudin M, Yulikov M, Veyre L, Berruyer P, Chaudhari S, Gajan D, Baudouin D, Cavaillès M, Vuichoud B, Bornet A, Jeschke G, Bodenhausen G, Lesage A, Emsley L, Jannin S, Thieuleux C, Copéret C (2017) Tailored Polarizing Hybrid Solids with Nitroxide Radicals Localized in Mesostructured Silica Walls. Helv Chim Acta 100:e1700101.

74. Capozzi A, Hyacinthe JN, Cheng T, Eichhorn TR, Boero G, Roussel C, van der Klink JJ, Comment A (2015) Photoinduced Nonpersistent Radicals as Polarizing Agents for X-Nuclei Dissolution Dynamic Nuclear Polarization. J Phys Chem C 119:22632-22639.

75. Capozzi A, Karlsson M, Petersen JR, Lerche MH, Ardenkjaer-Larsen JH (2018) Liquid-State ${ }^{13}$ C Polarization of 30\% through Photoinduced Nonpersistent Radicals. J Phys Chem C 122:7432-7443.

76. Capozzi A, Patel S, Gunnarsson CP, Marco-Rius I, Comment A, Karlsson M, Lerche MH, Ouari O, ArdenkjaerLarsen JH (2019) Efficient Hyperpolarization of U-(13) C-Glucose Using Narrow-Line UV-Generated Labile Free Radicals. Angew Chem Int Ed Engl 58:1334-1339.

77. Marco-Rius I, Cheng T, Gaunt AP, Patel S, Kreis F, Capozzi A, Wright AJ, Brindle KM, Ouari O, Comment A (2018) Photogenerated Radical in Phenylglyoxylic Acid for in Vivo Hyperpolarized (13)C MR with Photosensitive Metabolic Substrates. J Am Chem Soc 140:14455-14463.

78. Carravetta M, Johannessen OG, Levitt MH (2004) Beyond the T 1 Limit: Singlet Nuclear Spin States in Low Magnetic Fields. Phys Rev Lett 92:153003. 
79. Carravetta M, Levitt MH (2004) Long-Lived Nuclear Spin States in High-Field Solution NMR. J Am Chem Soc 126:6228-6229.

80. Vasos PR, Comment A, Sarkar R, Ahuja P, Jannin S, Ansermet J-P, Konter JA, Hautle P, van den Brandt B, Bodenhausen G (2009) Long-lived states to sustain hyperpolarized magnetization. Proc Natl Acad Sci 106:18469-18473.

81. Eills J, Cavallari E, Kircher R, Di Matteo G, Carrera C, Dagys L, Levitt MH, Ivanov K, Aime S, Reineri F, Münnemann K, Budker D, Buntkowsky G, Knecht S (2020) Singlet-Contrast Magnetic Resonance Imaging. doi: 10.26434/chemrxiv.12863639.v1

82. Dubroca T, Wi S, van Tol J, Frydman L, Hill S (2019) Large volume liquid state scalar Overhauser dynamic nuclear polarization at high magnetic field. Phys Chem Chem Phys 21:21200-21204.

83. Denysenkov V, Prandolini MJ, Gafurov M, Sezer D, Endeward B, Prisner TF (2010) Liquid state DNP using a 260 GHz high power gyrotron. Phys Chem Chem Phys 12:5786.

84. Bachert P, Brunner H, Hausser KH, Colpa JP (1984) Microwave-induced optical nuclear polarization (MI ONP) of benzophenone in dibromodiphenylether. Chem Phys 91:435-447.

85. Bargon J, Fischer H, Johnsen U (1967) Kernresonanz-Emissionslinien während rascher Radikalreaktionen. Z Für Naturforschung A 22:1551-1555.

86. Ward HRoy, Lawler RG (1967) Nuclear magnetic resonance emission and enhanced absorption in rapid organometallic reactions. J Am Chem Soc 89:5518-5519.

87. Iinuma M, Takahashi Y, Shaké I, Oda M, Masaike A, Yabuzaki T, Shimizu HM (2000) High Proton Polarization by Microwave-Induced Optical Nuclear Polarization at 77 K. Phys Rev Lett 84:171-174.

88. Eichhorn TR, Haag M, van den Brandt B, Hautle P, Wenckebach WTh (2013) High proton spin polarization with DNP using the triplet state of pentacene-. Chem Phys Lett 555:296-299.

89. Eichhorn TR, Niketic N, van den Brandt B, Filges U, Panzner T, Rantsiou E, Wenckebach WTh, Hautle P (2014) Proton polarization above $70 \%$ by DNP using photo-excited triplet states, a first step towards a broadband neutron spin filter. Nucl Instrum Methods Phys Res Sect Accel Spectrometers Detect Assoc Equip 754:10-14.

90. Tateishi K, Negoro M, Nishida S, Kagawa A, Morita Y, Kitagawa M (2014) Room temperature hyperpolarization of nuclear spins in bulk. Proc Natl Acad Sci 111:7527-7530.

91. Tateishi K, Negoro M, Kagawa A, Kitagawa M (2013) Dynamic Nuclear Polarization with Photoexcited Triplet Electrons in a Glassy Matrix. Angew Chem Int Ed 52:13307-13310.

92. Kouno H, Orihashi K, Nishimura K, Kawashima Y, Tateishi K, Uesaka T, Kimizuka N, Yanai N (2020) Triplet dynamic nuclear polarization of crystalline ice using water-soluble polarizing agents. Chem Commun 56:37173720.

93. Negoro M, Kagawa A, Tateishi K, Tanaka Y, Yuasa T, Takahashi K, Kitagawa M (2018) Dissolution Dynamic Nuclear Polarization at Room Temperature Using Photoexcited Triplet Electrons. J Phys Chem A 122:42944297.

94. Keshari KR, Kurhanewicz J, Macdonald JM, Wilson DM (2012) Generating contrast in hyperpolarized 13C MRI using ligand-receptor interactions. The Analyst 137:3427.

95. Can TV, Weber RT, Walish JJ, Swager TM, Griffin RG (2017) Frequency-Swept Integrated Solid Effect. Angew Chem Int Ed 56:6744-6748.

96. Henstra A, Dirksen P, Wenckebach WTh (1988) Enhanced dynamic nuclear polarization by the integrated solid effect. Phys Lett A 134:134-136.

97. Quan Y, van den Brandt B, Kohlbrecher J, Wenckebach WTh, Hautle P (2019) A transportable neutron spin filter. Nucl Instrum Methods Phys Res Sect Accel Spectrometers Detect Assoc Equip 921:22-26. 
98. King JP, Jeong K, Vassiliou CC, Shin CS, Page RH, Avalos CE, Wang H-J, Pines A (2015) Room-temperature in situ nuclear spin hyperpolarization from optically pumped nitrogen vacancy centres in diamond. Nat Commun 6:8965.

99. Comment A, van den Brandt B, Uffmann K, Kurdzesau F, Jannin S, Konter JA, Hautle P, Wenckebach WT, Gruetter R, van der Klink JJ (Aug) Principles of operation of a DNP prepolarizer coupled to a rodent MRI scanner. Appl Magn Reson 34:313-319.

100. Comment A, van den Brandt B, Uffmann K, Kurdzesau F, Jannin S, Konter JA, Hautle P, Wenckebach WTH, Gruetter R, van der Klink JJ (Oct) Design and performance of a DNP prepolarizer coupled to a rodent MRI scanner. Concepts Magn Reson Part B-Magn Reson Eng 31B:255-269.

101. Jahnig F, Kwiatkowski G, Dapp A, Hunkeler A, Meier BH, Kozerke S, Ernst M (2017) Dissolution DNP using trityl radicals at 7 T field. Phys Chem Chem Phys 19:19196-19204.

102. Jannin S, Comment A, Kurdzesau F, Konter JA, Hautle P, van den Brandt B, van der Klink JJ (Jun 28) A 140 GHz prepolarizer for dissolution dynamic nuclear polarization. J Chem Phys 128:241102 1-4.

103. Ardenkjaer-Larsen JH (2017) Cryogen-free dissolution dynamic nuclear polarization at $10 \mathrm{~T}$ (and other instrumental developments for dDNP). Asilomar, p 30

104. Bowen S, Rybalko O, Petersen JR, Ardenkaer-Larsen JH (2017) Multi-field cryogen free dissolution-DNP at 3.35, 6.70, and $10.05 \mathrm{~T}$.

105. Baudin M, Vuichoud B, Bornet A, Bodenhausen G, Jannin S (2018) A cryogen-consumption-free system for dynamic nuclear polarization at 9.4 T. J Magn Reson 294:115-121.

106. Bornet A, Pinon A, Jhajharia A, Baudin M, Ji X, Emsley L, Bodenhausen G, Ardenkjaer-Larsen JH, Jannin S (2016) Microwave-gated dynamic nuclear polarization. Phys Chem Chem Phys 18:30530-30535.

107. Batel M, Krajewski M, Weiss K, With O, Däpp A, Hunkeler A, Gimersky M, Pruessmann KP, Boesiger P, Meier BH, Kozerke S, Ernst M (2012) A multi-sample 94GHz dissolution dynamic-nuclear-polarization system. J Magn Reson 214:166-174.

108. Capozzi A, Kilund, J., Karlsson M, Lerche MH, Ardenkaer-Larsen JH (2020) Transportable hyperpolarized glucose samples: towards remote dissolution DNP. ISMRM 2020 New Front. Hyperpolarization Program Number 0693

109. Bowen S, Hilty C (2010) Rapid sample injection for hyperpolarized NMR spectroscopy. Phys Chem Chem Phys 12:5766-5770.

110. Kouřil K, Kouřilová H, Bartram S, Levitt MH, Meier B (2019) Scalable dissolution-dynamic nuclear polarization with rapid transfer of a polarized solid. Nat Commun 10:1733.

111. Gadian DG, Panesar KS, Perez Linde AJ, Horsewill AJ, Köckenberger W, Owers-Bradley JR (2012) Preparation of highly polarized nuclear spin systems using brute-force and low-field thermal mixing. Phys Chem Chem Phys 14:5397.

112. Peat DT, Hirsch ML, Gadian DG, Horsewill AJ, Owers-Bradley JR, Kempf JG (2016) Low-field thermal mixing in $\left[1-{ }^{13} \mathrm{C}\right]$ pyruvic acid for brute-force hyperpolarization. Phys Chem Chem Phys 18:19173-19182. 